\title{
Reduced Navier-Stokes equations near a flow boundary
}

\author{
M.S. Kilic ${ }^{\text {a }}$, G.B. Jacobs ${ }^{\text {b,c }}$, J.S. Hesthaven ${ }^{\text {b }}$, G. Haller ${ }^{\mathrm{c}, *}$ \\ ${ }^{a}$ Department of Mathematics, MIT, 77 Massachusetts Ave., Cambridge, MA 02139, USA \\ ${ }^{\mathrm{b}}$ Division of Applied Mathematics, Brown University, Providence, RI, 02912,USA \\ ${ }^{\mathrm{c}}$ Department of Mechanical Engineering, MIT, 77 Massachusetts Ave., Cambridge, MA 02139, USA
}

Received 4 August 2005; received in revised form 17 March 2006; accepted 5 April 2006

Available online 6 May 2006

Communicated by U. Frisch

\begin{abstract}
We derive a hierarchy of PDEs for the leading-order evolution of wall-based quantities, such as the skin-friction and the wall-pressure gradient, in two-dimensional fluid flows. The resulting Reduced Navier-Stokes (RNS) equations are defined on the boundary of the flow, and hence have reduced spatial dimensionality compared to the Navier-Stokes equations. This spatial reduction speeds up numerical computations and makes the equations attractive candidates for flow-control design. We prove that members of the RNS hierarchy are well-posed if appended with boundaryconditions obtained from wall-based sensors. We also derive the lowest-order RNS equations for three-dimensional flows. For several benchmark problems, our numerical simulations show close finite-time agreement between the solutions of RNS and those of the full Navier-Stokes equations. (c) 2006 Elsevier B.V. All rights reserved.
\end{abstract}

Keywords: Reduced-order models; Navier-Stokes equations; Flow control

\section{Introduction}

\subsection{Background and motivation}

The approximation of Navier-Stokes flows near a no-slip boundary was apparently first discussed in detail by Perry and Chong [11], who developed a procedure for finding the Taylor coefficients of a velocity field expanded at a boundary point. By this procedure, one can construct velocity models that are polynomials in terms of the distance from the point of expansion. The models are dynamically consistent up to any desired order, but depend on properties imposed a priori on the velocity derivatives at the wall.

Danielson and Ottino [3] used the above procedure to construct a system of ODEs for the Taylor coefficients of a velocity field at a no-slip boundary point. The ODE system becomes finite-dimensional upon truncation of the Taylor expansion; Danielson and Ottino showed that even low-order truncations may lead to ODEs with a strange attractor, a hallmark of Eulerian turbulence.

Recently, Bewley and Protas [12] proposed a less restrictive Taylor-expansion of the velocity in terms of the normal distance from the boundary. For two-dimensional flows, this procedure yields a single-variable Taylor-expansion with coefficients depending on the location along the boundary. Bewley and Protas showed that under appropriate conditions, the expansion converges in the vicinity of the wall. In addition, for incompressible flows, all Taylor coefficients can be expressed in terms of time- and walltangential derivatives of the wall shear (skin friction) and the wall pressure.

With the availability of accurate skin-friction and pressure sensor-arrays, the results in [12] enable local velocity reconstruction from wall-based measurements. This offers a promising tool for practical flow control, where the impact of the controller must be evaluated from wall sensors. Feedback control, however, requires more than just an observation of the output: a model for the

\footnotetext{
* Corresponding author. Tel.: +1 6174523064 .

E-mail address: ghaller@mit.edu (G. Haller).
} 


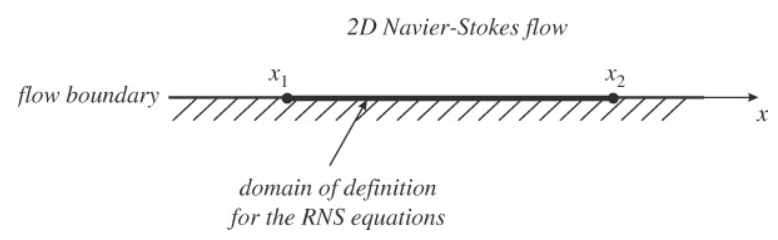

Fig. 1. Domain of definition for the RNS equations.

evolution of the flow is also crucial. The Bewley-Protas results offer hope that, at least near the wall, such models are reducible to depend only on the skin friction and wall pressure.

Further underlying the need for such reduced flow models, typical performance objectives in flow-control are often phrased directly in terms of skin friction and wall pressure, not velocity. Examples include pressure-recovery enhancement in diffusers and surface-drag reduction on submarines. The former aims to maximize the integral of the wall-pressure gradient; the latter to minimize the integral of the skin friction. In both cases, a qualitative prediction for the evolution of the underlying quantity is more beneficial than a highly accurate but complex numerical model.

\subsection{Main results}

Motivated by the above, here we study how the dynamics of wall-based quantities, such as the wall-shear $\tau$ and the wall-pressure gradient $\gamma$, can be modelled and predicted in two-dimensional Navier-Stokes flows. Our main result is a hierarchy of models, the Reduced Navier-Stokes $(R N S)$ equations, that describe the evolution of the above quantities at different levels of accuracy. Since the RNS equations are defined on the flow boundary, they only have one spatial dimension, the wall coordinate $x$. This dimensional reduction results in computation times that are significantly shorter than those of direct Navier-Stokes simulations.

Solving the RNS equations requires updated boundary conditions for $\tau$ and $\gamma$ at two $x$-locations, $x_{1}$ and $x_{2}$ (see Fig. 1). Thus, $x_{1}$ and $x_{2}$ must either be points with a priori known velocity derivatives (e.g., corner points), or must lie within distributed skin-friction and wall-pressure sensor arrays. In either case, the RNS equations provide qualitative prediction for the time-evolution of $\tau(x, t)$ and $\gamma(x, t)$ over the spatial interval $\left[x_{1}, x_{2}\right]$. The prediction necessarily deteriorates over time; solving the RNS equations over longer times therefore requires periodic re-initialization by sampling $\tau(x, t)$ and $\gamma(x, t)$ from sensors distributed over $\left[x_{1}, x_{2}\right]$.

We derive three members of the RNS hierarchy explicitly; these evolution equations are obtained from cubic, quartic, and quintic truncations of the Taylor expansion of the wall-tangential velocity component. We prove that these three RNS equations and all higher-order RNS systems are well-posed, i.e., admit unique solutions that depend continuously on the initial data. We also derive the lowest-order RNS equation for three-dimensional flows, and discuss the relevance of the two-dimensional RNS equations in select flow-control problems.

We present evidence for the accuracy of the RNS equations by comparing their numerical solution to classic solutions of the Navier-Stokes equations. These classic solutions include a viscous channel flow, the Blasius boundary layer solution, viscous flow near a stagnation point, and an oscillating flow over an infinite plate. We finally compare the direct numerical simulation of a lid-driven cavity flow to that of the RNS equations. In all cases, we observe close quantitative agreement on short to intermediate timescales, and qualitative accuracy over longer timescales.

\section{RNS equations for two-dimensional flows}

Consider the two-dimensional Navier-Stokes equations

$$
\begin{aligned}
& \partial_{t} u+u_{x} u+u_{y} v=-\frac{1}{\rho} p_{x}+v\left(u_{x x}+u_{y y}\right), \\
& \partial_{t} v+v_{x} u+v_{y} v=-\frac{1}{\rho} p_{y}+v\left(v_{x x}+v_{y y}\right),
\end{aligned}
$$

where $(u(x, y, t), v(x, y, t))$ is a velocity field satisfying the incompressibility condition

$$
u_{x}+v_{y}=0,
$$

and the no-slip boundary condition

$$
u(x, 0, t)=v(x, 0, t)=0
$$

at the $y=0$ boundary. In (1), $p(x, y, t)$ denotes the pressure, and $v$ and $\rho$ are the kinematic viscosity and the density of the fluid. 
We seek to understand the evolution of the skin-friction field

$$
\tau(x, t)=\rho \nu u_{y}(x, 0, t),
$$

and the wall-pressure-gradient field

$$
\gamma(x, t)=p_{x}(x, 0, t)=\rho v u_{y y}(x, 0, t) .
$$

As auxiliary variables to be used later, we also introduce

$$
\sigma(x, t)=\rho \nu u_{y y y}(x, 0, t), \quad \lambda(x, t)=\rho \nu u_{y y y y}(x, 0, t), \quad \eta(x, t)=\rho v u_{y y y y y}(x, 0, t) .
$$

With the above variables, the Taylor expansion of $u(x, y, t)$ near the $y=0$ boundary can be written as

$$
u(x, y, t)=\frac{1}{v \rho}\left[\tau(x, t) y+\frac{1}{2} \gamma(x, t) y^{2}+\frac{1}{6} \sigma(x, t) y^{3}+\frac{1}{24} \lambda(x, t) y^{4}+\frac{1}{120} \eta(x, t) y^{5}+\mathcal{O}\left(y^{6}\right)\right]
$$

Subtracting the $x$-derivative of the second equation in (1) from the $y$-derivative of the first equation, we obtain the vorticity-transport equation

$$
\partial_{t}\left(u_{y}-v_{x}\right)+u\left(u_{x y}-v_{x x}\right)+v\left(u_{x x}+u_{y y}\right)=v\left(2 u_{x x y}+u_{y y y}-v_{x x x}\right),
$$

from which we shall derive approximate expressions for the evolution of the Taylor coefficients in (5).

\subsection{The cubic RNS equations}

Setting $y=0$ in Eq. (6) yields

$$
\tau_{t}=v\left(2 \tau_{x x}+\sigma\right) .
$$

Differentiating (6) with respect to $y$ and using the incompressibility condition (2) leads to

$$
\begin{aligned}
& \partial_{t}\left(u_{x x}+u_{y y}\right)+u_{y}\left(u_{x y}-v_{x x}\right)+u\left(u_{x y y}+u_{x x x}\right)-u_{x}\left(u_{x x}+u_{y y}\right)+v\left(u_{x x y}+u_{y y y}\right) \\
& \quad=v\left(2 u_{x x y y}+u_{y y y y}+u_{x x x x}\right) .
\end{aligned}
$$

After setting $y=0$ in (8), we obtain

$$
\gamma_{t}+\frac{1}{v \rho} \tau \tau_{x}=v\left(2 \gamma_{x x}+v \rho u_{y y y}\right)
$$

Finally, differentiating (8) with respect to $y$, we find that

$$
\begin{aligned}
& \partial_{t}\left(u_{x x y}+u_{y y y}\right)+u_{y y}\left(u_{x y}-v_{x x}\right)+u_{y}\left(u_{x y y}+u_{x x x}\right)+u_{y}\left(u_{x y y}+u_{x x x}\right)+u\left(u_{x y y y}+u_{x x x y}\right)-u_{x y}\left(u_{x x}+u_{y y}\right) \\
& -2 u_{x}\left(u_{y y y}+u_{x x y}\right)+v\left(u_{y y y y}+u_{x x y y}\right)=v\left(2 u_{x x y y y}+u_{y y y y y}+u_{x x x x y}\right) .
\end{aligned}
$$

Setting $y=0$ in this last equation, we obtain

$$
\left[\partial_{t}\left(u_{x x y}+u_{y y y}\right)+2 u_{y} u_{y y x}\right]_{y=0}=v\left(2 u_{x x y y y}+u_{y y y y y}+u_{x x x x y}\right)_{y=0} .
$$

At the same time, the second $x$-derivative of (6) taken at $y=0$ is

$$
\left(\partial_{t} u_{x x y}\right)_{y=0}=v\left(2 u_{x x x x y}+u_{x x y y y}\right)_{y=0} .
$$

Combining (11) and (12) gives

$$
\sigma_{t}+\frac{2}{v \rho} \tau \gamma_{x}=v\left(\sigma_{x x}+v \rho u_{y y y y y}-\tau_{x x x x}\right) .
$$

We now assume that the velocity component $u(x, y, t)$ is well approximated by its third-order Taylor expansion near the $y=0$ boundary; in other words, we truncate the expansion (5) at cubic order. Under this approximation, Eqs. (7), (9) and (13) yield the cubic RNS equations

$$
\begin{aligned}
\tau_{t} & =2 \nu \tau_{x x}+v \sigma \\
\gamma_{t} & =2 \nu \gamma_{x x}-\frac{1}{\nu \rho} \tau \tau_{x}, \\
\sigma_{t} & =v \sigma_{x x}-\nu \tau_{x x x x}-\frac{2}{\nu \rho} \tau \gamma_{x} .
\end{aligned}
$$


To this nonlinear system of PDEs, we add the boundary and initial conditions

$$
\begin{aligned}
& \tau(0, t)=T_{0}(t), \quad \tau(L, t)=T_{1}(t), \quad \tau\left(x, t_{0}\right)=\tau^{0}(x), \\
& \gamma(0, t)=G_{0}(t), \quad \gamma(L, t)=G_{1}(t), \quad \gamma\left(x, t_{0}\right)=\gamma^{0}(x), \\
& \sigma(0, t)=S_{0}(t), \quad \sigma(L, t)=S_{1}(t), \quad \sigma\left(x, t_{0}\right)=\sigma^{0}(x) .
\end{aligned}
$$

The initial and boundary conditions for $\tau$ may be obtained from distributed skin-friction sensors; the same conditions for $\gamma$ can be measured by distributed pressure sensors. Realistic sensors do not exist for measuring $\sigma$ directly, but distributed skin-friction sensors can be used to find the values of $\sigma$ from the relations

$$
\sigma(0, t)=\dot{T}_{0}(t)-2 v \tau_{x x}(0, t), \sigma(L, t)=\dot{T}_{L}(t)-2 v \tau_{x x}(L, t), \sigma\left(x, t_{0}\right)=\dot{\tau}\left(x, t_{0}\right)-2 v \tau_{x x}^{0}(x) .
$$

The solvability of the cubic RNS equations (14) is guaranteed by the following result.

Theorem 2.1. The cubic RNS equations (14) with the boundary and initial conditions (15) are well-posed: they admit unique solutions $(\tau, \gamma, \sigma)$ with continuous dependence on initial data in the function space $H^{3}[0, L] \times H^{2}[0, L] \times H^{1}[0, L]$.

\section{Proof. See Appendix A.1.}

The advantage of the cubic RNS equations (14) is that they are defined on the one-dimensional spatial domain $[0, L]$, as opposed to the Navier-Stokes equations (1) that are defined on a two-dimensional domain. System (14), however, cannot be solved independently: its time-dependent boundary conditions are to be obtained from pressure- and skin-friction sensors at the boundary. In addition, a distributed skin-friction and pressure measurement at $t=0$ is necessary to identify the initial condition.

Since the system (14) is obtained from a Taylor-series truncation of the original velocity field, it will only approximate true Navier-Stokes solutions for finite times. As a result, (14) must be periodically re-initialized for its solutions to stay accurate. Our main motivation is controller design, for which the simplicity and the short-term predictive power of (14) is more important than its long-term accuracy.

\subsection{The quartic RNS equations}

For increased accuracy, we now derive a higher-order approximation for the evolution of velocity derivatives at the wall. Differentiating (10) with respect to $y$, we obtain

$$
\begin{gathered}
\partial_{t}\left(u_{x x y y}+u_{y y y y}\right)-u_{y y y} v_{x x}+u_{y y}\left(2 u_{x y y}+2 u_{x x x}\right)+3 u_{y}\left(u_{x y y y}+u_{x x x y}\right)+u\left(u_{x y y y y}+u_{x x x y y}\right)-u_{x y y} u_{x x} \\
-3 u_{x y} u_{x x y}-2 u_{x y} u_{y y y}-3 u_{x}\left(u_{y y y y}+u_{x x y y}\right)+v\left(u_{y y y y y}+u_{x x y y y}\right)=v\left(2 u_{x x y y y}+u_{y y y y y y}+u_{x x x x y y}\right) .
\end{gathered}
$$

Setting $y=0$ in this equation gives

$$
\begin{aligned}
& {\left[\partial_{t}\left(u_{x x y y}+u_{y y y y}\right)+2 u_{y y} u_{x y y}+3 u_{y}\left(u_{x y y y}+u_{x x x y}\right)-3 u_{x y} u_{x x y}-2 u_{x y} u_{y y y}\right]_{y=0}} \\
& \quad=v\left(2 u_{x x y y y y}+u_{y y y y y y}+u_{x x x y y}\right)_{y=0} .
\end{aligned}
$$

Next, we differentiate (8) twice with respect to $x$ and set $y=0$ to obtain

$$
\left[\partial_{t} u_{y y x x}+3 u_{x y} u_{x x y}+u_{y} u_{x x x y}\right]_{y=0}=v\left(2 u_{x x x x y y}+u_{x x y y y y}+u_{x x x x x x}\right)_{y=0} .
$$

Subtracting (18) from (17) then gives

$$
\left[\partial_{t} u_{y y y y}+2 u_{y y} u_{x y y}+u_{y}\left(3 u_{x y y y}+2 u_{x x x y}\right)-6 u_{x y} u_{x x y}-2 u_{x y} u_{y y y}\right]_{y=0}=v\left(u_{x x y y y y}+u_{y y y y y y}-u_{x x x x y y}\right)_{y=0},
$$

or, equivalently,

$$
\partial_{t} \lambda+\frac{2}{v \rho} \gamma \gamma_{x}+\frac{1}{v \rho} \tau\left(3 \sigma_{x}+2 \tau_{x x x}\right)-\frac{6}{v \rho} \tau_{x} \tau_{x x}-\frac{2}{v \rho} \tau_{x} \sigma=v\left(\lambda_{x x}+v \rho u_{y y y y y y}-\gamma_{x x x x}\right) .
$$

We now assume that the velocity component $u(x, y, t)$ is well approximated by its fourth-order Taylor expansion near the boundary, i.e., we truncate the expansion (5) at quartic order. Under this approximation, Eqs. (7), (9) and (13) can be summarized in the quartic RNS equations

$$
\begin{aligned}
\tau_{t} & =2 v \tau_{x x}+v \sigma, \\
\gamma_{t} & =2 \nu \gamma_{x x}+v \lambda-\frac{1}{v \rho} \tau \tau_{x}, \\
\sigma_{t} & =v \sigma_{x x}-v \tau_{x x x x}-\frac{2}{v \rho} \tau \gamma_{x},
\end{aligned}
$$




$$
\lambda_{t}=v \lambda_{x x}-v \gamma_{x x x x}-\frac{2}{v \rho} \gamma \gamma_{x}-\frac{1}{v \rho} \tau\left(3 \sigma_{x}+2 \tau_{x x x}\right)+\frac{6}{v \rho} \tau_{x} \tau_{x x}+\frac{2}{v \rho} \tau_{x} \sigma,
$$

with the initial and boundary conditions

$$
\begin{array}{ll}
\tau(0, t)=T_{0}(t), & \tau(L, t)=T_{1}(t), \quad \tau\left(x, t_{0}\right)=\tau^{0}(x), \\
\gamma(0, t)=G_{0}(t), & \gamma(L, t)=G_{1}(t), \quad \gamma\left(x, t_{0}\right)=\gamma^{0}(x), \\
\sigma(0, t)=S_{0}(t), & \sigma(L, t)=S_{1}(t), \quad \sigma\left(x, t_{0}\right)=\sigma^{0}(x), \\
\lambda(0, t)=L_{0}(t), & \sigma(L, t)=L_{1}(t), \quad \lambda\left(x, t_{0}\right)=\lambda^{0}(x) .
\end{array}
$$

The boundary and initial conditions for $\lambda$ can again be obtained from distributed skin-friction and pressure measurements:

$$
\begin{aligned}
& \lambda(0, t)=\left[\dot{G}_{0}(t)-2 v \gamma_{x x}(0, t)+T_{0}(t) \tau_{x}(0, t) /(v \rho)\right] / v, \\
& \lambda(L, t)=\left[\dot{G}_{1}(t)-2 v \gamma_{x x}(L, t)+T_{1}(t) \tau_{x}(L, t) /(v \rho)\right] / v, \\
& \lambda\left(x, t_{0}\right)=\dot{\gamma}\left(x, t_{0}\right)-2 v \gamma_{x x}^{0}(x)+\tau^{0}(x) \tau_{x}^{0}(x) /(v \rho) .
\end{aligned}
$$

The solvability of the quartic RNS equations (14) is guaranteed by the following result.

Theorem 2.2. The quartic RNS equations (19) with the boundary and initial conditions (20) are well-posed on the function space $H^{4}[0, L] \times H^{3}[0, L] \times H^{2}[0, L] \times H^{1}[0, L]$.

Proof. See Appendix A.2

Note that the evolution of the pressure gradient and the skin friction remain independent in Stokes flows even after the addition of higher-order terms. The same conclusion will hold for any higher-order RNS equation. In those higher-order equations, the only further change is the appearance of linear coupling terms to higher-order $y$-derivatives of $u$. This suggests that the first robust enough model for both the skin friction and the pressure gradient is the quartic model.

\subsection{Higher-order RNS equations}

In order to derive an $n$th order approximation for the evolution of flow-derivatives at the wall, we subtract the second $x$-derivative of the order $n-2$ RNS equations from the (n-3)-order $y$-derivative of Eq. (10). Setting $y=0$ in the resulting equation and neglecting $y$-derivatives of $u$ higher in order than $n$, we obtain the $n$ th-order RNS equations. For example, the quintic RNS equations take the form

$$
\begin{aligned}
& \tau_{t}=2 v \tau_{x x}+v \sigma, \\
& \gamma_{t}=2 v \gamma_{x x}+v \lambda-\frac{1}{v \rho} \tau \tau_{x}, \\
& \sigma_{t}=v \sigma_{x x}-v \tau_{x x x x}+v \eta-\frac{2}{v \rho} \tau \gamma_{x}, \\
& \lambda_{t}=v \lambda_{x x}-v \gamma_{x x x x}-\frac{2}{v \rho} \gamma \gamma_{x}-\frac{1}{v \rho} \tau\left(3 \sigma_{x}+2 \tau_{x x x}\right)+\frac{6}{v \rho} \tau_{x} \tau_{x x}+\frac{2}{v \rho} \tau_{x} \sigma, \\
& \eta_{t}=v \eta_{x x}+v \tau_{x x x x x x}+\frac{1}{v \rho}\left[\left(5 \lambda+8 \gamma_{x x}\right) \tau_{x}-5 \gamma\left(\sigma_{x}+\tau_{x x x}\right)-\tau\left(4 \lambda_{x}+3 \gamma_{x x x}\right)+5 \tau_{x x} \gamma_{x}\right],
\end{aligned}
$$

with appropriate boundary and initial conditions.

Our regularity results extend to the solutions of higher-order RNS equations:

Theorem 2.3. For any $n \geq 3$, the nth-order RNS equations are well-posed on the function space $H^{n}[0, L] \times \cdots \times H^{1}[0, L]$.

Proof. See Appendix A.2.

\section{RNS equations for three-dimensional flows}

Although the focus of the present paper is two-dimensional flows, we briefly discuss here how analogous equations can be derived in three dimensions. We start with the three-dimensional Navier-Stokes equations

$$
u_{t}+u_{x} u+u_{y} v+u_{z} w=-\frac{1}{\rho} p_{x}+v\left(u_{x x}+u_{y y}+u_{z z}\right)
$$




$$
\begin{aligned}
& v_{t}+v_{x} u+v_{y} v+v_{z} w=-\frac{1}{\rho} p_{y}+v\left(v_{x x}+v_{y y}+v_{z z}\right) \\
& w_{t}+w_{x} u+w_{y} v+w_{z} w=-\frac{1}{\rho} p_{z}+v\left(w_{x x}+w_{y y}+w_{z z}\right)
\end{aligned}
$$

where $(u(x, y, z, t), v(x, y, z, t), w(x, y, z, t))$ is a velocity field satisfying the incompressibility condition

$$
u_{x}+v_{y}+w_{z}=0
$$

and the no-slip boundary condition

$$
u(x, y, 0, t)=0, \quad v(x, y, 0, t)=0 .
$$

In (22), $p(x, y, z, t)$ denotes the pressure, and $v$ and $\rho$ are the kinematic viscosity and the density of the fluid.

The skin-friction field is now defined as

$$
\left(\tau^{1}(x, y, t), \tau^{2}(x, y, t)\right)=\left.\left(\rho \nu u_{z}, \rho \nu v_{z}\right)\right|_{z=0},
$$

and the wall-pressure-gradient field is equal to

$$
\left(\gamma^{1}(x, y, t), \gamma^{2}(x, y, t)\right)=\left(p_{x}(x, y, 0, t), p_{y}(x, y, 0, t)\right)=\left.\left(\rho \vee u_{z z}, \rho \nu v_{z z}\right)\right|_{z=0} .
$$

As auxiliary variables to be used later, we also introduce

$$
\begin{aligned}
& \left(\lambda^{1}, \lambda^{2}\right)=\left.\left(\rho \nu u_{z z z}, \rho \nu v_{z z z}\right)\right|_{z=0}, \\
& \left(\sigma^{1}, \sigma^{2}\right)=\left.\left(\rho \nu u_{z z z z}, \rho \nu v_{z z z z}\right)\right|_{z=0} .
\end{aligned}
$$

With these variables, the Taylor expansion of $u(x, y, z, t)$ and $v(x, y, z, t)$ at the boundary $z=0$ can be written as

$$
\begin{aligned}
& u(x, y, z, t)=\frac{1}{\rho v}\left[\tau^{1}(x, y, t) z+\frac{1}{2} \gamma^{1}(x, y, t) z^{2}+\frac{1}{6} \sigma^{1}(x, y, t) z^{3}+\frac{1}{24} \lambda^{1}(x, y, t) z^{4}+O\left(z^{5}\right)\right] \\
& v(x, y, z, t)=\frac{1}{\rho v}\left[\tau^{2}(x, y, t) z+\frac{1}{2} \gamma^{2}(x, y, t) z^{2}+\frac{1}{6} \sigma^{2}(x, y, t) z^{3}+\frac{1}{24} \lambda^{2}(x, y, t) z^{4}+O\left(z^{5}\right)\right] .
\end{aligned}
$$

As we show in Appendix B, the truncation of the expansion (23) at cubic order leads to the three-dimensional cubic RNS equation

$$
\begin{aligned}
& \tau_{t}^{1}= 2 v \tau_{x x}^{1}+v \tau_{y y}^{1}+v \tau_{x y}^{2}+v \sigma^{1}, \\
& \tau_{t}^{2}=2 v \tau_{y y}^{2}+v \tau_{x x}^{2}+v \tau_{x y}^{1}+v \sigma^{2}, \\
& \gamma_{t}^{1}=v\left(2 \gamma_{x x}^{1}+\gamma_{y y}^{1}+\lambda^{1}+\gamma_{x y}^{2}\right)-\frac{1}{v \rho}\left(\tau^{1} \tau_{x}^{1}-2 \tau_{y}^{1} \tau^{2}-\tau^{1} \tau_{y}^{2}\right), \\
& \gamma_{t}^{2}=v\left(2 \gamma_{y y}^{2}+\gamma_{x x}^{2}+\lambda^{2}+\gamma_{x y}^{2}\right)-\frac{1}{v \rho}\left(\tau^{2} \tau_{y}^{2}-2 \tau_{x}^{2} \tau^{1}-\tau^{2} \tau_{x}^{1}\right), \\
& \sigma_{t}^{1}=-v\left[3 \tau_{x x x x}^{1}+3 \tau_{y y x x}^{1}+\sigma_{x x}^{1}+3 \tau_{x x x y}^{2}+3 \tau_{y y y x}^{2}+2 \sigma_{x y}^{2}-\sigma_{y y}^{1}\right] \\
&+\frac{1}{v \rho}\left[-2 \gamma_{x}^{1} \tau^{1}-3 \gamma_{y}^{1} \tau^{2}-3 \tau_{y}^{1} \gamma^{2}+3 \gamma^{1} \tau_{y}^{2}+\tau^{1} \gamma_{y}^{2}-3 \tau_{x x}^{1} \tau^{1}\right] \\
&+\frac{1}{v \rho}\left[-3 \tau_{x y}^{2} \tau^{1}+3 \tau_{x y}^{1} \tau^{2}+3 \tau_{y y}^{2} \tau^{2}+3\left(\tau_{x}^{1}\right)^{2}+6 \tau_{x}^{1} \tau_{y}^{2}+3\left(\tau_{y}^{2}\right)^{2}\right], \\
& \sigma_{t}^{2}=-v\left[3 \tau_{y y y}^{2}+3 \tau_{y y x x}^{2}+\sigma_{y y}^{2}+3 \tau_{x y y y}^{1}+3 \tau_{y x x}^{1}+2 \sigma_{x y}^{1}-\sigma_{x x}^{2}\right] \\
&+\frac{1}{v \rho}\left[-2 \gamma_{y}^{2} \tau^{2}-3 \gamma_{x}^{2} \tau^{1}-3 \tau_{x}^{2} \gamma^{1}+3 \gamma^{2} \tau_{x}^{1}+\tau^{2} \gamma_{x}^{1}-3 \tau_{y y}^{2} \tau^{2}\right] \\
&+\frac{1}{v \rho}\left[-3 \tau_{x y}^{1} \tau^{2}+3 \tau_{x y}^{2} \tau^{1}+3 \tau_{x x}^{1} \tau^{1}+3\left(\tau_{x}^{1}\right)^{2}+6 \tau_{x}^{1} \tau_{y}^{2}+3\left(\tau_{y}^{2}\right)^{2}\right] .
\end{aligned}
$$

\section{The $\tau-\bar{p}$ formulation with application to flow control}

Here we discuss the relevance of the RNS equations in flow modelling and control. First, we recall that all derivatives of an incompressible velocity field at a no-slip wall can be expressed in terms of the skin friction, pressure, their wall gradients, and their time derivatives (see Bewley and Protas [12]). This implies that (14) can be re-written as a two-dimensional set of PDEs for $\tau$ and $\gamma$. 


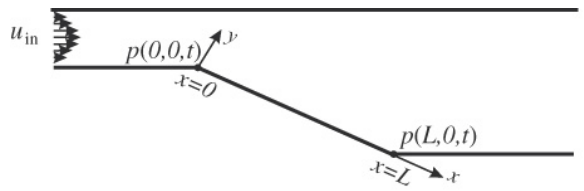

(a)

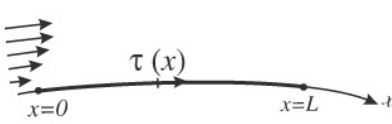

(b)

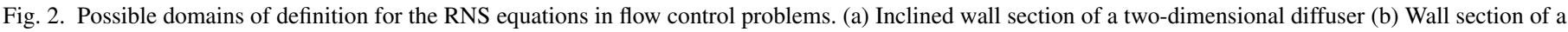
two-dimensional streamlined body.

Indeed, differentiating the first equation of (14) in time gives

$$
\begin{aligned}
\tau_{t t} & =2 v \tau_{x x t}+v \sigma_{t} \\
& =2 v \tau_{x x t}+v\left[v\left(\frac{1}{v} \tau_{x x t}-2 \tau_{x x x x}\right)-v \tau_{x x x x}-\frac{2}{v \rho} \tau \gamma_{x}\right],
\end{aligned}
$$

which, combined with the second equation of (14), gives the system of PDEs

$$
\begin{aligned}
& \tau_{t t}=3 v \tau_{x x t}-3 v^{2} \tau_{x x x x}-\frac{2}{\rho} \tau \gamma_{x}, \\
& \gamma_{t}=2 v \gamma_{x x}-\frac{1}{v \rho} \tau \tau_{x} .
\end{aligned}
$$

Integrating the second equation of (25) with respect to $x$ over the interval $[0, x]$ and introducing the pressure difference $\bar{p}(x, t)=p(x, t)-p(0, t)$, we obtain the $\tau-\bar{p}$ form of the cubic RNS system:

$$
\begin{aligned}
\tau_{t t} & =3 v \tau_{t x x}-3 v^{2} \tau_{x x x x}-\frac{2}{\rho} \tau \bar{p}_{x x}, \\
\bar{p}_{t} & =2 v \bar{p}_{x x}+\frac{1}{v \rho}\left[\int_{0}^{x} \tau_{x}^{2}(s, t) \mathrm{d} s-\tau^{2}\right]+\frac{1}{v \rho} \tau^{2}(0, t)-2 v \bar{p}_{x x}(0, t) .
\end{aligned}
$$

This formulation is preferable over (14) if the pressure and skin-friction boundary conditions at $x=0$ are trivial. For instance, if $x=0$ coincides with the location of a vertical no-slip wall (i.e., there is a corner at $x=0$ ), then we have

$$
\tau(0, t)=0, \quad p_{x x}(0, t)=0,
$$

where the second relation follows from the Navier-Stokes equations (1). In this case, the $\tau-\bar{p}$ form of the cubic RNS equation simplifies to

$$
\begin{aligned}
& \tau_{t t}=3 v \tau_{t x x}-3 v^{2} \tau_{x x x x}-\frac{2}{\rho} \tau \bar{p}_{x x}, \\
& \bar{p}_{t}=2 v \bar{p}_{x x}+\frac{1}{v \rho}\left[\int_{0}^{x} \tau_{x}^{2}(s, t) \mathrm{d} s-\tau^{2}\right] .
\end{aligned}
$$

Setting the correct boundary conditions for this equation only requires time-resolved skin-friction and pressure measurements at $x=L$.

The $\tau-\bar{p}$ formulation (26) (or (27)) offers a lowest-order approximation for the evolution of the skin friction and the wall-pressure gradient. Such approximations are useful in flow control problems where the cost function only depends on $\tau$ or $\bar{p}$.

For instance, if $x \in[0, L]$ varies on a wall segment connecting the inlet and the outlet of a diffuser (see Fig. 2(a)), then losses in the diffuser are measured by the pressure recovery

$$
C_{p}(t)=\frac{\bar{p}(L, 0, t)}{\frac{1}{2} \rho u_{\mathrm{in}}^{2}(t)},
$$

with $u_{\text {in }}$ denoting the mean inflow velocity. The losses will be minimal if $C_{p}$ is maximal. Assuming constant-in-time inflow conditions, $C_{p}(t)$ will be maximal if $\bar{p}(L, 0, t)$ is maximal. Thus, to minimize losses in the diffuser, the time-average of the cost function

$$
c_{p}(t)=\bar{p}(L, 0, t)
$$

is to be maximized. 
Another relevant example is surface drag reduction over the boundary section $[0, L]$ of a streamlined body (see Fig. 2(b)). Since the surface drag force is the integral of the wall shear along the wall, minimizing the average of the cost function

$$
c_{\tau}(t)=\int_{0}^{L} \tau(x, t) \mathrm{d} x,
$$

minimizes surface drag over $[0, L]$.

In deriving the RNS hierarchy, we have assumed no-slip boundary conditions on the $[0, L]$ wall segment. Therefore, the domain of definition of the RNS equations cannot contain wall-based fluidic actuators performing blowing or suction. Such actuators can be placed outside $[0, L]$ - preferably close to $x=0$ or $x=L$ - to control the boundary conditions for the RNS equations.

\section{Numerical study of the RNS equations}

Here we show by examples that the cubic, quartic, and quintic RNS solutions approximate true Navier-Stokes solutions well over characteristic time intervals. The examples include a channel flow, a Blasius boundary layer flow, a viscous flow near a stagnation point, an oscillating flow above an infinite plane, and a lid-driven cavity flow.

In the first four examples, the Navier-Stokes equations admit exact or simplified steady-state solutions. We use these steadystate solutions as initial conditions for the RNS equations, and monitor how the solutions obtained this way deviate from the exact steady-state solutions. In our last example, we compare direct numerical solutions of the Navier-Stokes and the RNS equations. In this case, the boundary conditions for the RNS equations are a priori known: the normal derivatives of the $u$-velocity are zero at the corners of the lid-driven cavity.

We use a Chebyshev spectral scheme for the spatial approximation of (14), (19) and (21). We choose this method for its high accuracy and for its ability to treat Dirichlet boundary conditions (see Canuto et al. [2]). For temporal integration, we use a secondorder implicit Crank-Nicholson scheme (Canuto et al. [2]) combined with a Newton-Krylov solver (Kelley [7]). We present our simulation results using the nondimensional (convective) time

$$
\bar{t}=t u_{0} / L_{0},
$$

where $u_{0}$ is a characteristic velocity and $L_{0}$ is a characteristic length.

\section{Channel flow}

\section{Analytical solution}

For a laminar flow between two parallel plates at distance $L$, the Navier-Stokes equations (1) simplify to

$$
v u_{y y}=\frac{1}{\rho} p_{x},
$$

if we assume that the vertical velocity $v$ vanishes, and $u$ does not vary with $x$. Consequently, $p_{x} \equiv p_{x}^{0}$ is a constant.

Integrating (28) with respect to $y$ and using the symmetry condition $u_{y}(x, L / 2)=0$ leads to

$$
u_{y}(x, y)=\frac{1}{v \rho} p_{x}^{0}(y-L / 2) .
$$

Integrating once more and using the no-slip condition $u=0$ at $y=0$ yields

$$
u(x, y)=\frac{1}{2 v \rho} p_{x}^{0}\left(y^{2}-L y\right) .
$$

Setting $u(x, L / 2)=u_{\max }$ then gives

$$
p_{x}^{0}=-\frac{8 u_{\max } v \rho}{L^{2}},
$$

thus the solution of (28) can be written as

$$
u(x, y)=-4 u_{\max }\left[\left(\frac{y}{L}\right)^{2}-\frac{y}{L}\right] .
$$

\section{Validation}

From (32), we obtain

$$
\tau_{\text {exact }}=-\frac{4 \rho \nu u_{\max }}{L}, \quad \gamma_{\text {exact }}=-\frac{8 u_{\max } v \rho}{L^{2}}, \quad \sigma_{\text {exact }}=0, \quad \lambda_{\text {exact }}=0 .
$$



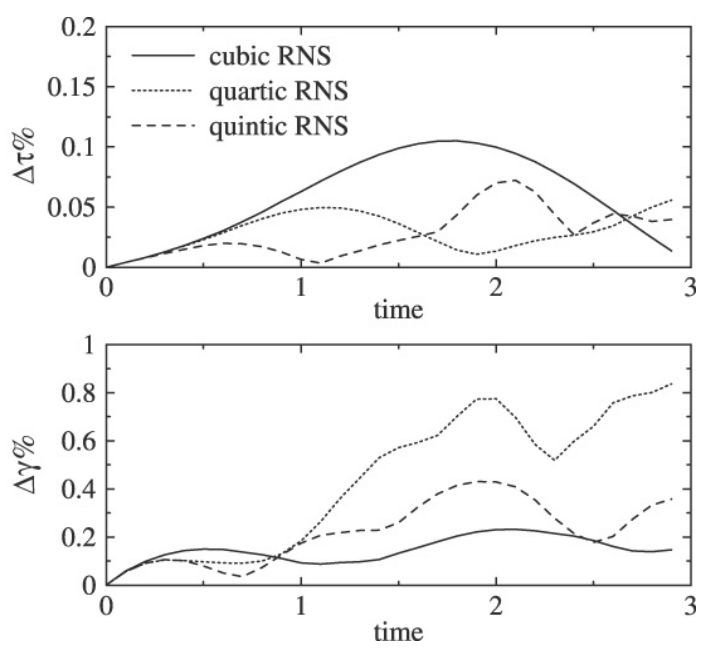

Fig. 3. RNS simulations for $\tau$ and $\gamma$ in the perturbed channel flow with $\epsilon=10^{-3}$. The percentage error for $\tau$ is obtained as $\Delta \tau \%=\frac{100}{N}\left[\sum_{i=1}^{N}\left|\tau-\tau_{i}\right|\right] / \max _{i}\left|\tau_{i}\right|$ where $\tau_{1}, \ldots, \tau_{N}$ are the $\tau$ values at $N$ gridpoints $x_{1}, \ldots, x_{N} \in[0,1]$; the definition of $\Delta \gamma \%$ is similar.

As a direct substitution into (14) and (19) shows, (33) is an exact solution of the cubic and quartic RNS equations. This was to be expected, because the solution (32) is exactly equal to its cubic and quartic Taylor-expansion in $y$.

In practice, however, initial conditions for the RNS equations are determined from sensors and hence are subject to measurement errors. We emulate such errors by selecting the perturbed initial condition

$$
\tau=\tau_{\text {exact }}[1+\epsilon \sin (2.0 \pi x)], \quad \epsilon \ll 1,
$$

in our RNS simulation instead of the exact steady-state given in (33). We select the parameters $u_{\max }=1 \mathrm{~m} / \mathrm{s}, v=0.01 \mathrm{~m}^{2} / \mathrm{s}$, and $\rho=1.0 \mathrm{~kg} / \mathrm{m}^{3}$ in our simulations.

At this low Reynolds number ( $\left.R e=u_{\max } L / v=10\right)$, the full Navier-Stokes equations damp out the perturbation in (34) and the flow returns to steady state. By contrast, the cubic RNS equations produce slowly growing oscillations about the steady state for the perturbed initial condition (34). Positive news on the growth is that it is weak: it does not exceed the order of the perturbation on timescales of $\mathcal{O}(1)$. This can be seen in Fig. 3, where the percentage errors in $\tau$ and $\gamma$ are plotted for different RNS equations.

As seen from Fig. 3, the quartic RNS equations limit the error growth better for $\tau$. The quintic RNS equations (21) provide further improvement for $\bar{t}<1$, but for larger times, the error grows faster. For $\bar{t}>5$, the error exceeds the order of the perturbation, and hence the RNS equations have to be re-initialized. Interestingly, increasing the order of the RNS equation results in growing errors for $\gamma$; the errors, however, still remain below $1 \%$ for times up to $\bar{t}=3$.

Despite the growing errors, qualitative accuracy of the RNS equations persists for extended times. Specifically, qualitative correctness holds up to $\bar{t}=7$ in the cubic and quartic case, and up to $\bar{t}=5$ in the quintic case (see Fig. 4).

Finally, we illustrate continuous dependence on initial conditions for the cubic and quartic RNS equations, as established in Theorems 2.1 and 2.2. Fig. 5 shows that decreasing the size of the perturbation parameter $\epsilon$ in (34) indeed leads to slower error growth.

\section{Blasius boundary layer}

\section{Similarity solution}

Here we consider a similarity solution to the zero-pressure-gradient boundary layer over a flat plate accredited to Blasius (White [13]). For this flow, the Prandtl boundary-layer equations give

$$
u(x, y)=U f^{\prime}(\xi), \quad|v(x, y)| \ll|u(x, y)|,
$$

where $U$ is a reference velocity outside the boundary layer, prime denotes differentiation with respect to the variable $\xi=\frac{y}{x} \sqrt{\frac{U x}{v}}$, and $f$ satisfies the ODE

$$
2 f^{\prime \prime \prime}+f f^{\prime}=0
$$

The boundary conditions for $f$ are $f(0)=f^{\prime}(0)=0$ and $f^{\prime}(\infty) \rightarrow 1$. Solving (36) with these boundary conditions gives $f^{\prime \prime}(0)=0.33206$. From (35), we obtain

$$
\tau_{\text {exact }}(x)=\rho U \sqrt{\frac{\nu U}{x}} f^{\prime \prime}(0), \quad \gamma_{\text {exact }}=0, \quad \sigma_{\text {exact }}=0, \quad \lambda_{\text {exact }}(x)=\frac{\rho U^{3}}{2 v x^{2}} f^{\prime \prime 2}(0), \quad \eta_{\text {exact }}=0 .
$$



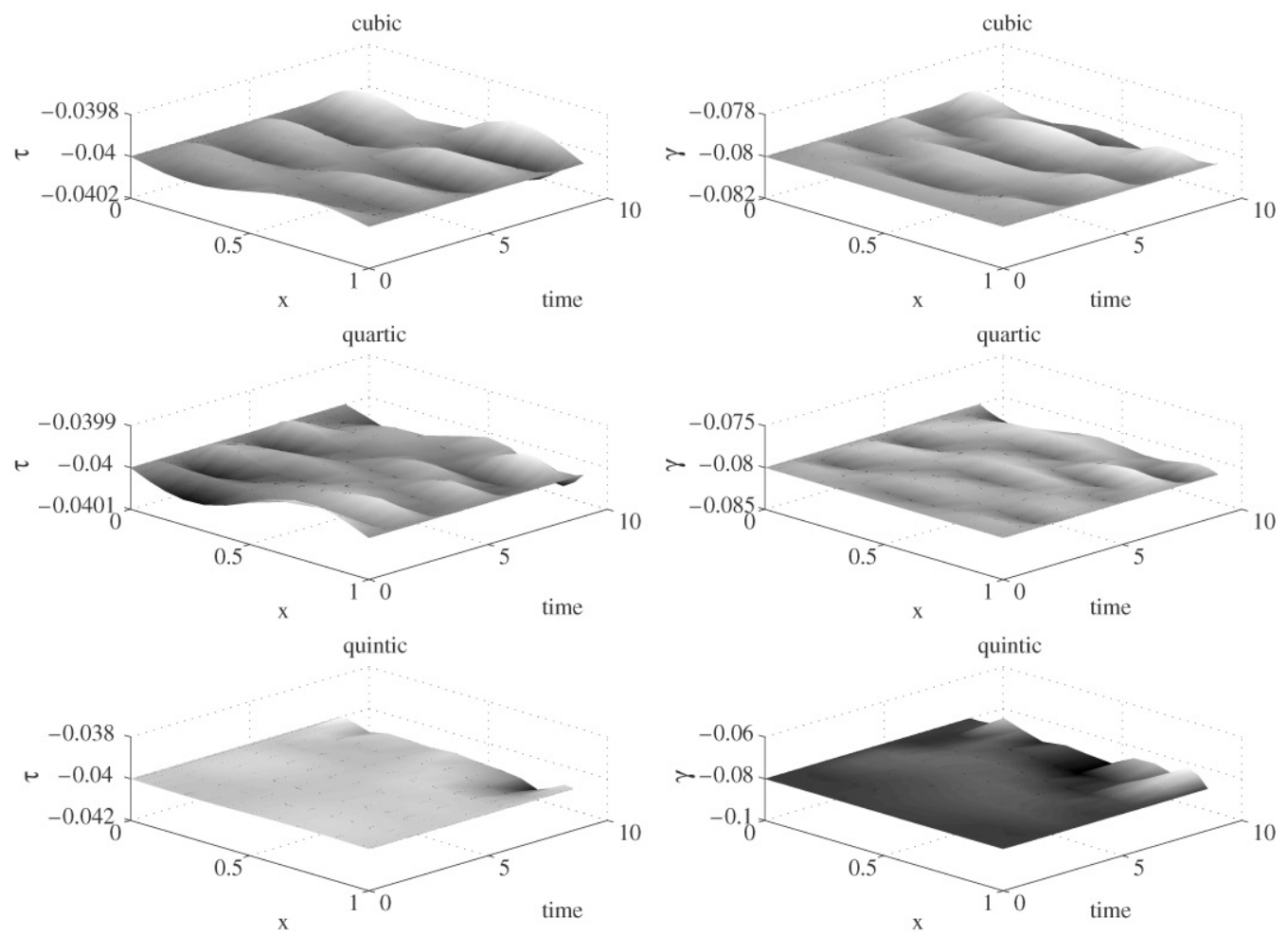

Fig. 4. Evolution of the $\tau$ and $\gamma$ profiles in the cubic, quartic, and quintic RNS equations for the channel flow. At the nondimensional time $\bar{t}=0$, the profiles agree with the exact solution.
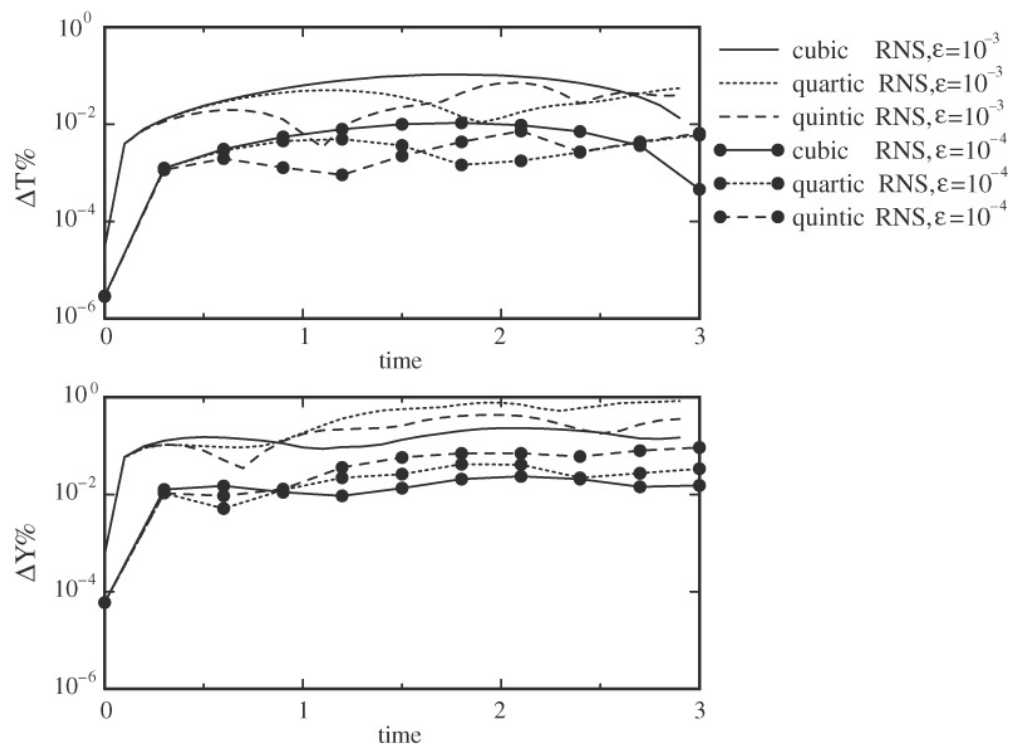

Fig. 5. Evolution of the $\tau$ and $\gamma$ profiles for $\epsilon=10^{-3}$ and $\epsilon=10^{-4}$ in the cubic and quartic RNS equations for the channel flow.

\section{Validation}

We use (37) to obtain initial and boundary conditions for our simulation of the RNS equations (14), (19) and (21). We solve these equations over the spatial interval $x \in[1,2]$ with $U=1 \mathrm{~m} / \mathrm{s}, v=10^{-3} \mathrm{~m}^{2} / \mathrm{s}$, and $\rho=1.0 \mathrm{~kg} / \mathrm{m}^{3}$. For these parameter values, the Reynolds number is $R e=10^{3}$, at which the boundary-layer equations are a good approximation to the Navier-Stokes equations, and hence can be compared to corresponding RNS solutions. Fig. 6 shows such a comparison with the percentage error in $\tau$ and $\gamma$ for the cubic, quartic, and quintic RNS equations.

The error growth is faster than in the previous channel flow example, but still remains below $1 \%$ up to one convective time unit. Notably, the error in the quartic RNS is now significantly less than in the cubic RNS. The quintic RNS, however, is less accurate than the quartic, because the terms neglected in the boundary layer approximation vanish in the quartic RNS, but not in the quintic RNS. 

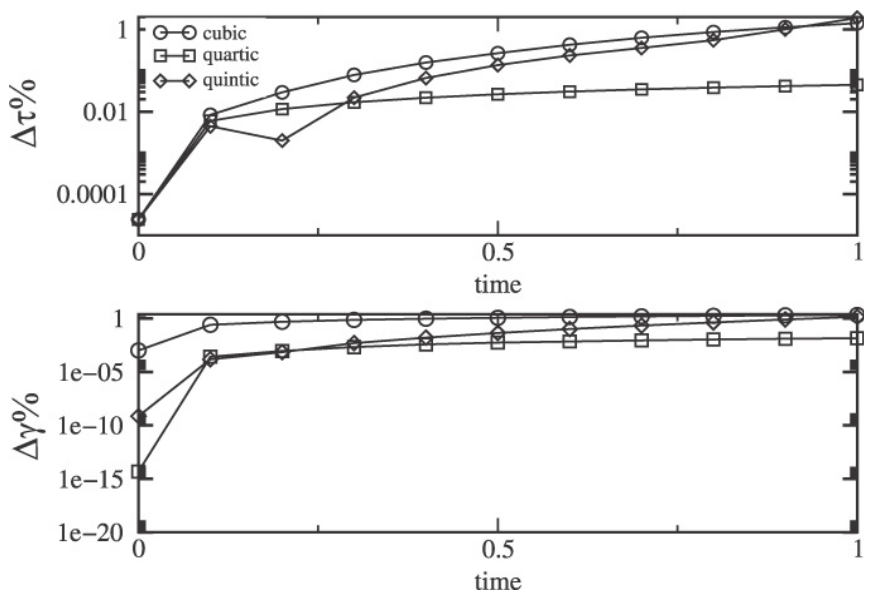

Fig. 6. Percentage of error in $\tau+$ and $\gamma$ as a function of time for the Blasius profile.
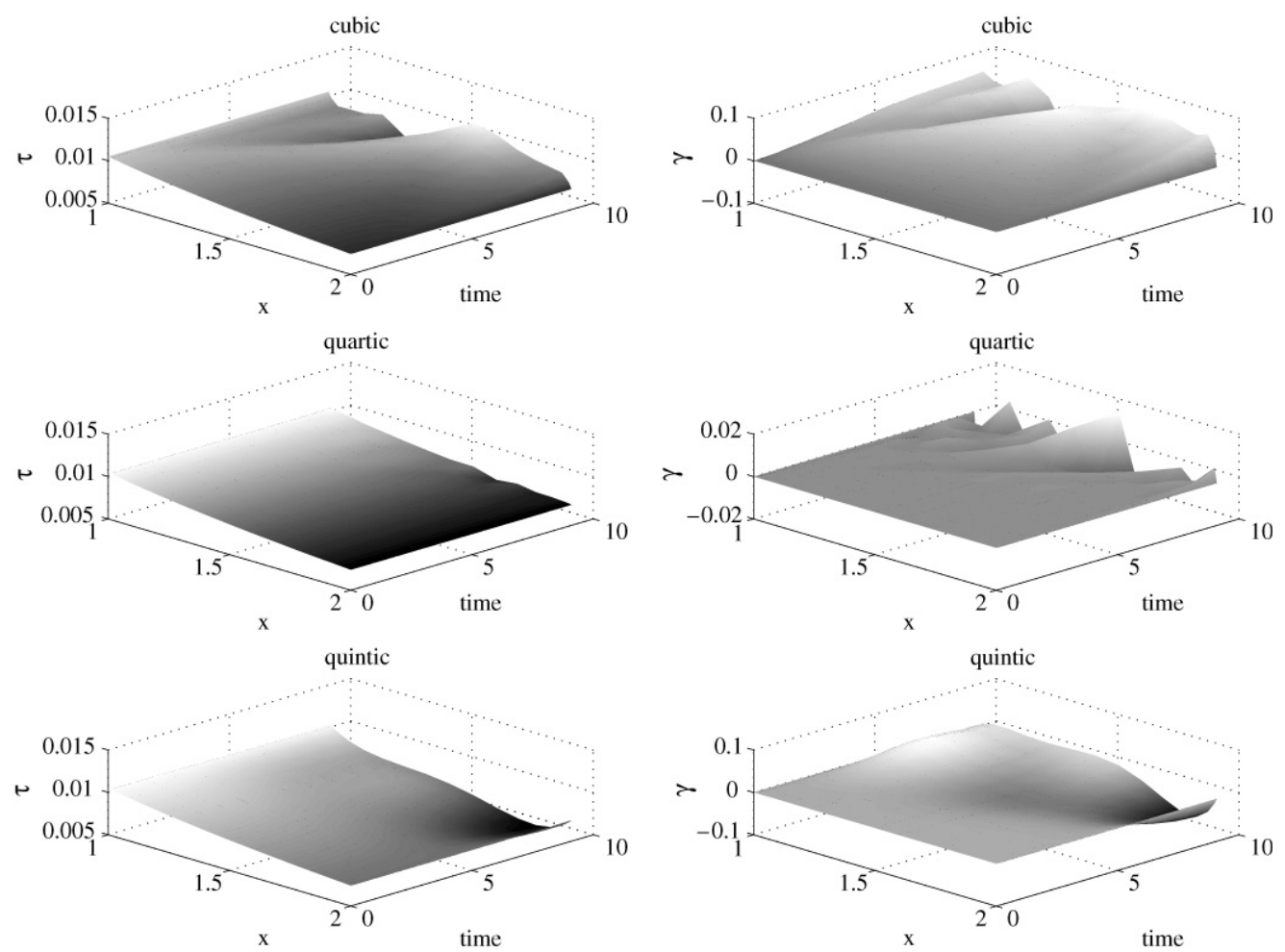

Fig. 7. Evolution of the $\tau$ and $\gamma$ profiles in the cubic, quartic, and quintic RNS equations for the Blasius boundary layer flow. At the nondimensional time $\bar{t}=0$, the profiles agree with the exact solution.

For this example, the qualitative accuracy of the RNS systems is evidenced by Fig. 7. Even the cubic RNS solutions remain qualitatively accurate up to $\bar{t}=2$ convective time units, but the quartic RNS systems perform significantly better.

\section{Viscous flow near a stagnation point}

\section{Similarity solution}

For a viscous stagnation point flow, the Navier-Stokes equations (1) yield the similarity solution

$$
u(x, y)=B x F(\xi),
$$

where $F$ satisfies the ODE

$$
F^{\prime \prime \prime}+F F^{\prime \prime}+1-F^{\prime 2}=0,
$$

with prime denoting differentiation with respect to $\xi=y \sqrt{\frac{B}{v}}$. The parameter $B$ is proportional to the ratio of a reference velocity $U$ and a reference length $L$ (White [13]). 

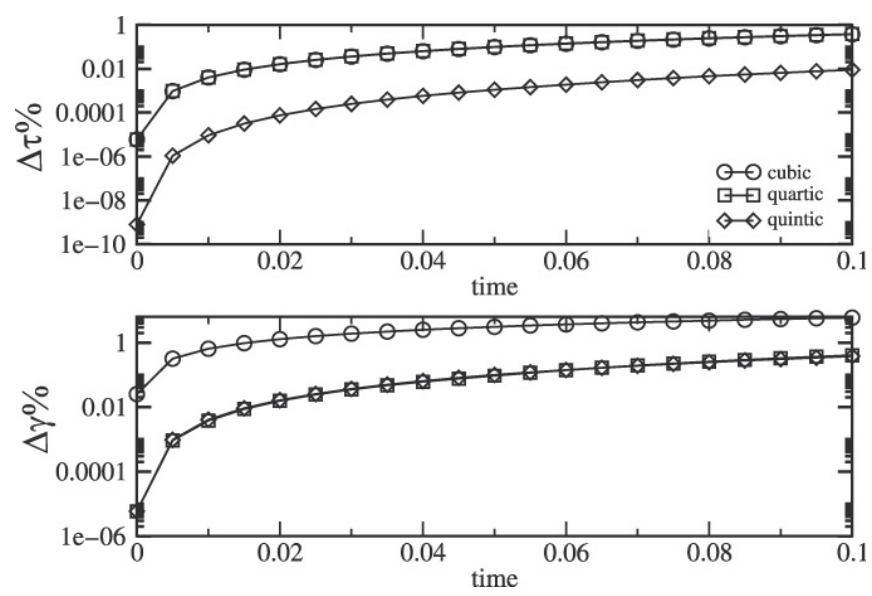

Fig. 8. Percentage of error in $\tau$ and $\gamma$ as a function of time for the viscous stagnation point flow.

The boundary conditions for (39) are $F(0)=F^{\prime}=0$ and $F^{\prime}(\infty)=1$. Solving (39) numerically with these boundary conditions yields $F^{\prime \prime}(0)=1.23259$. From the velocity expression (38), we find that

$$
\begin{aligned}
& \tau_{\text {exact }}(x)=B \rho \sqrt{v B} F^{\prime \prime}(0) x, \quad \gamma_{\text {exact }}(x)=-B^{2} \rho x, \quad \sigma_{v}(x)=0.0 \text {, } \\
& \lambda_{\text {exact }}(x)=\frac{B^{4} \rho}{v}\left[F^{\prime \prime}(0)\right]^{2} x, \quad \eta_{\text {exact }}(x)=-2 \sqrt{\frac{B}{v}} \frac{B^{4} \rho}{v} F^{\prime \prime}(0) x .
\end{aligned}
$$

\section{Validation}

For the numerical solution of the RNS equations, we obtain initial and boundary conditions from (40). We solve the equations on the spatial domain $x \in[0,1 \mathrm{~m}]$ with $B=1 \mathrm{~s}^{-1}$ and $v=10^{-2} \mathrm{~m}^{2} / \mathrm{s}$; the corresponding Reynolds number is $R e=100$, which is well within the validity of the laminar stagnation flow approximation.

Fig. 8 again shows limited error growth. In this simulation, however, the $1 \%$ error bar is reached in 0.1 nondimensional time units, indicating faster error growth than in earlier examples. While the quartic RNS is an order of magnitude more accurate for $\gamma$ than the cubic RNS, the two sets of equations perform equally well for $\tau$. The quintic model shows a much improved estimation for the evolution of $\tau$.

As for qualitative accuracy, we show the time evolution of the $\tau$ and $\gamma$ profiles in Fig. 9. In all cases, qualitative closeness to the approximate analytic solution is maintained for convective times up to $\bar{t}=1$.

\section{Fluid oscillation over an infinite plate}

\section{Analytical solution}

For an unsteady parallel laminar flow, the Navier-Stokes equations reduce to

$$
u_{t}=-\frac{1}{\rho} p_{x}+v u_{y y}
$$

The pressure gradient can only be a function of time for this flow, and hence can be absorbed into the velocity by a change of variables leading to a homogeneous diffusion equation (White [13]).

For an oscillating wall with $u(0, t)=U_{0} \cos \omega t$ with zero velocity far from the wall, the velocity field is of the form $u(y, t)=f(y) \mathrm{e}^{\mathrm{i} \omega t}$. Substitution into the above-mentioned diffusion equation leads to $f=\mathrm{e}^{-\eta} \cos (\omega t-\eta)$, where $\eta=y \sqrt{\frac{\omega}{2 v}}$. The real part of $u(y, t)$ is then given by

$$
u(y, t)=U_{0} \mathrm{e}^{-\eta} \cos (\omega t-\eta) .
$$

With (42) at hand, we find that

$$
\begin{aligned}
& \tau_{\text {exact }}(x)=-\rho \nu B[\cos \omega t-\sin \omega t], \\
& \gamma_{\text {exact }}(x)=-2 \rho \nu B^{2} \sin \omega t, \\
& \sigma_{\text {exact }}(x)=2 \rho \nu B^{3}[\sin \omega t-\cos \omega t], \\
& \lambda_{\text {exact }}(x)=-4 \rho \nu B^{4} \cos \omega t, \\
& \eta_{\text {exact }}(x)=4 \rho \nu B^{5}[\cos \omega t-\sin \omega t],
\end{aligned}
$$

where $B=\sqrt{\omega /(2 v)}$. Note that all the above quantities are independent of $x$. 

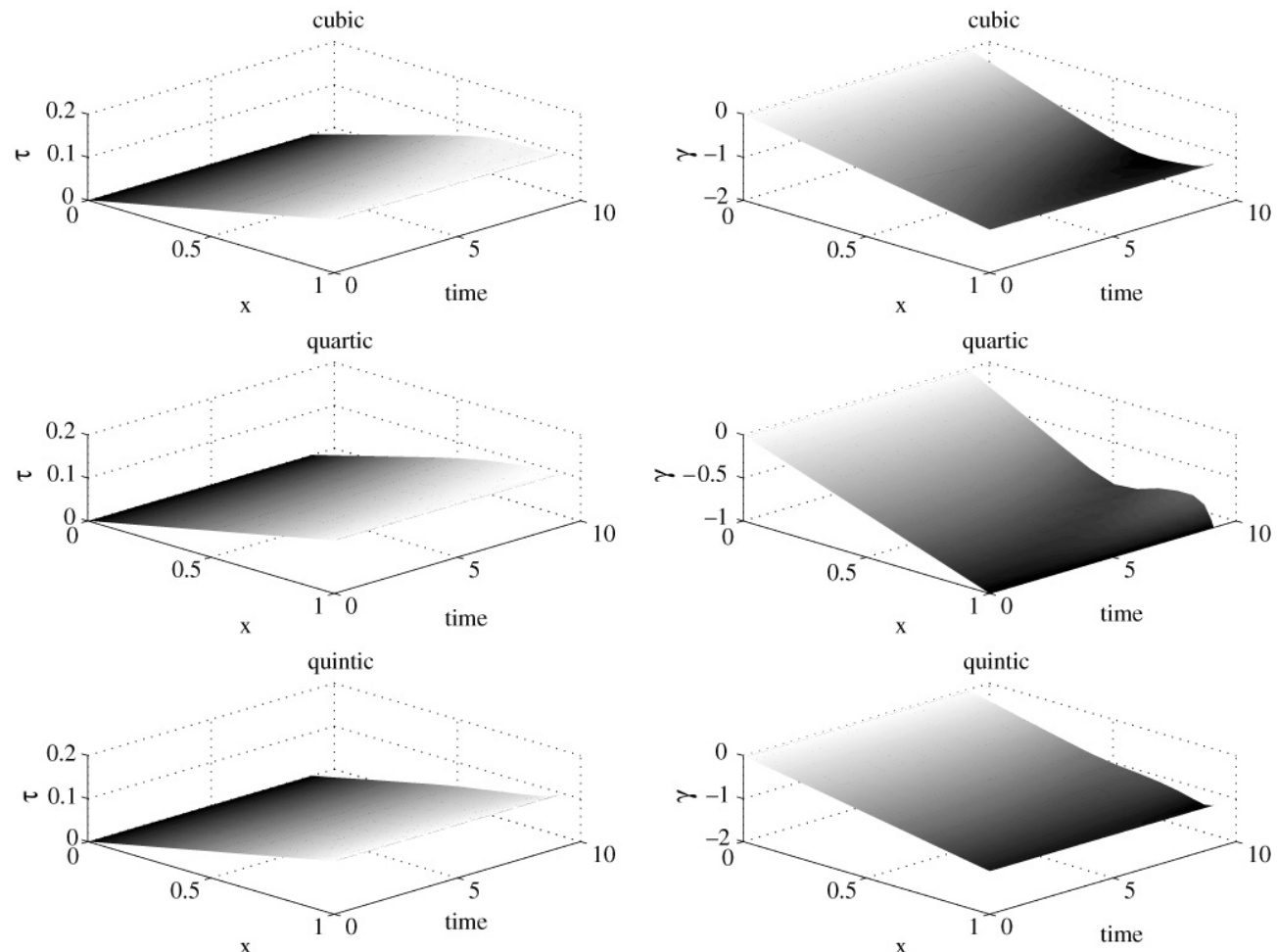

Fig. 9. Evolution of the $\tau$ and $\gamma$ profiles in the cubic, quartic, and quintic RNS equations for the stagnation point flow. At the nondimensional time $\bar{t}=0$, the profiles agree with the exact solution.
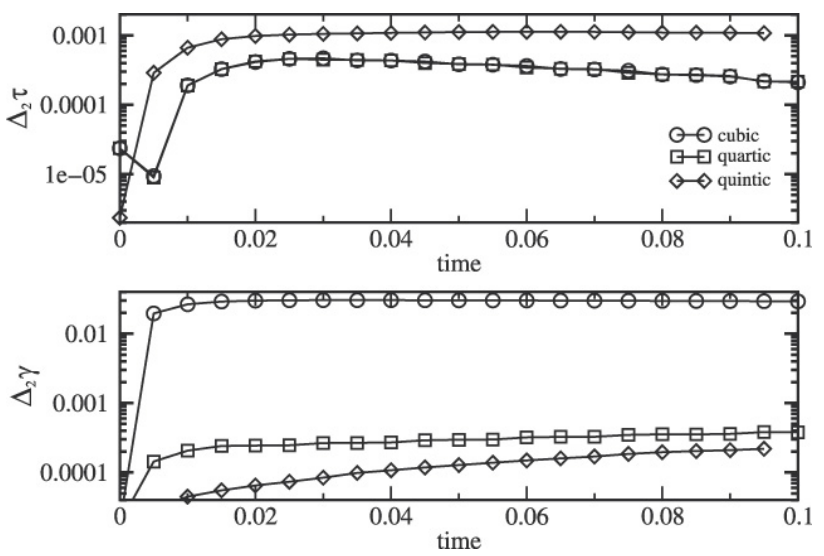

Fig. 10. $L_{2}$ error in $\tau$ and $\gamma$ as a function of time for flow oscillating over an infinite plate.

\section{Validation}

Again, (43) provide initial and boundary conditions for our RNS simulations. We select $U_{0}=1 \mathrm{~m} / \mathrm{s}, v=10 \mathrm{~m}^{2} / \mathrm{s}$, and $\omega=\pi 1 / \mathrm{s}$; we solve the cubic, quartic, and quintic RNS equations for $0 \leq x \leq 1 \mathrm{~m}$. The corresponding Reynolds number is $R e=0.1$, which is consistent with the Stokes-flow approximation present in (41). Fig. 10 shows that the $L_{2}$ error in $\tau$ and $\gamma$ remains below $3 \%$ for up to 0.1 nondimensional time unit. In addition, the quartic model predicts $\gamma$ two orders of magnitude more accurately than the cubic model.

In this example, we observe qualitative correctness for the RNS systems on long timescales. Shown in Fig. 11, the $\tau$ and $\gamma$ profiles stay qualitatively close to the exact solution for up to four nondimensional time units; the errors appear uniformly bounded for all times.

\subsection{Steady and unsteady lid-driven cavity flow}

As our final example, we use direct numerical simulation of a lid-driven cavity to test the accuracy of the RNS equations. The simulations are performed with a staggered-grid multidomain spectral method (Kopriva [8] and Jacobs et al. [9]). The computational model consists of a square whose upper boundary is a lid moving at a constant speed. 

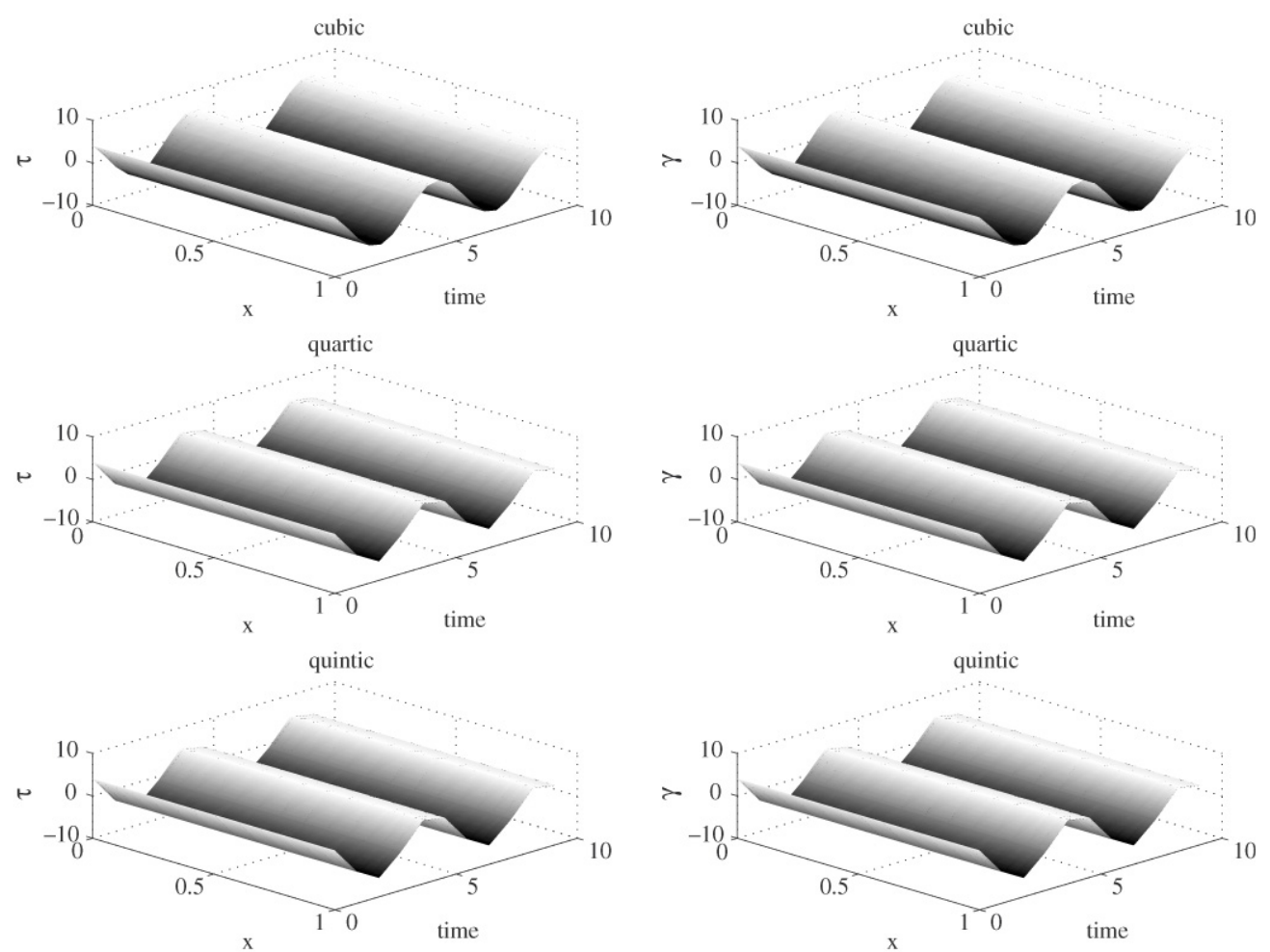

Fig. 11. Evolution of the $\tau$ and $\gamma$ profiles in the cubic, quartic, and quintic RNS equations for the stagnation point flow. At the nondimensional time $\bar{t}=0$, the profiles agree with the exact solution.

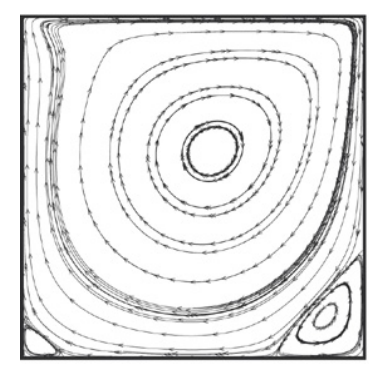

(a)

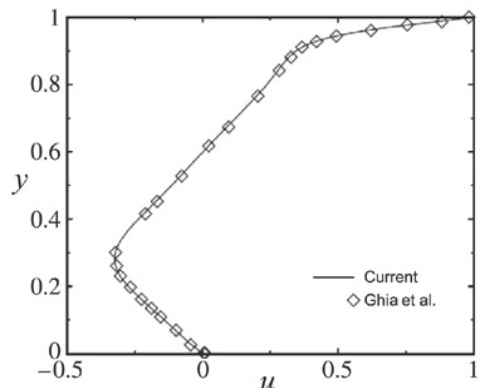

(b)

Fig. 12. (a) Streamlines at the steady state of a lid-driven cavity flow at $R e=400$. (b) Comparison of a $u$-velocity profile along the midplane for the steady state of a lid-driven cavity at $R e=400$.

The Reynolds number $R e=400$ of the flow is based on the velocity of the lid and the length of the square's side. The flow is started from a quiescent state that develops into a steady state. The steady-state solution is characterized by three vortices, as shown by the streamlines in Fig. 12(a). This steady-state solution agrees with previously published results by Ghia et al. [4], as seen in Fig. 12(b).

At the steady state, we evaluate the $y$-derivatives of the velocity to be used as initial conditions for the RNS equations. The boundary conditions for the RNS equations at $x=0 \mathrm{~m}$ and $x=L=1 \mathrm{~m}$ are zero because of the zero derivatives at the corners of the cavity.

Figs. 13 and 14 show that up to time $\bar{t}=1$, the skin friction $\tau$ remains within $1 \%$ of its steady state value for both the cubic and the quartic RNS equations. Over the same time period, $\gamma$ shows errors in the order of $10 \%$. Qualitative correctness holds up to time $\bar{t}=2$ for $\gamma$, and up to $\bar{t}=3$ for $\tau$.

Fig. 15 compares $\tau$ for the initial unsteady cavity flow. The velocity gradients at $\bar{t}=5.0$ are used as initial conditions for the RNS equations. At later times, the computed $\tau$ values from the RNS equations are compared with the simulation. We find that at time $\bar{t}=5.1$, the RNS equations compare reasonably with the simulation. Although at $\bar{t}=5.2$, the RNS equations show substantial deviation from the Navier-Stokes simulation, the $\tau$ profile is still qualitatively correct. With initial conditions re-set at $\bar{t}=5.1$, the RNS equations again remain accurate for times of order 0.1; qualitative accuracy again persists somewhat longer, but is lost by $\bar{t}=5.5$. 

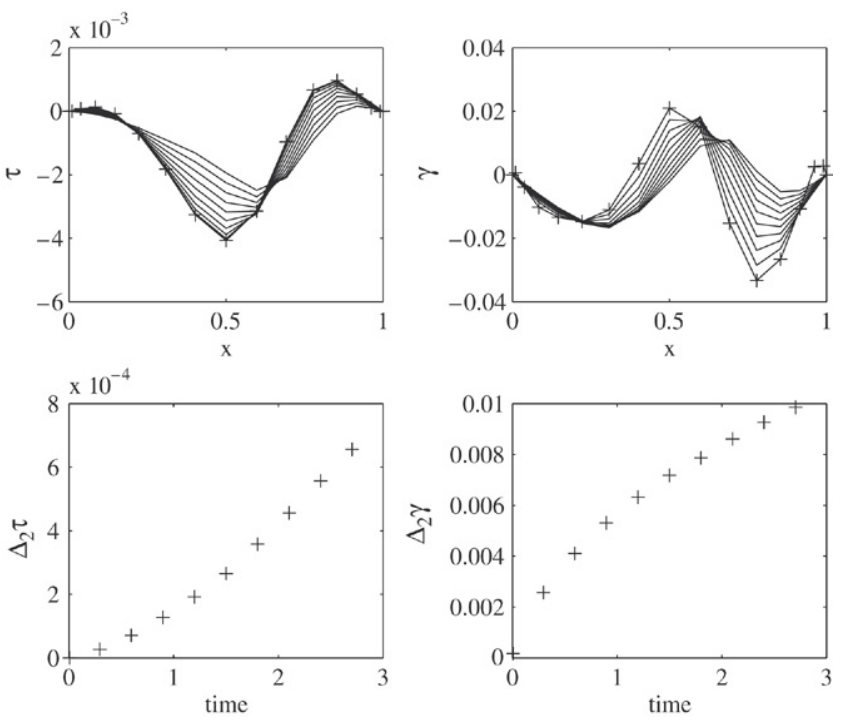

Fig. 13. Errors in the cubic RNS simulation of the lid-driven cavity flow. The (absolute) errors $\Delta \tau$ and $\Delta \gamma$ are shown as functions of the location for different times; the $L_{2}$-errors $\Delta_{2} \tau$ and $\Delta_{2} \gamma$ are shown as functions of the nondimensionalized time.
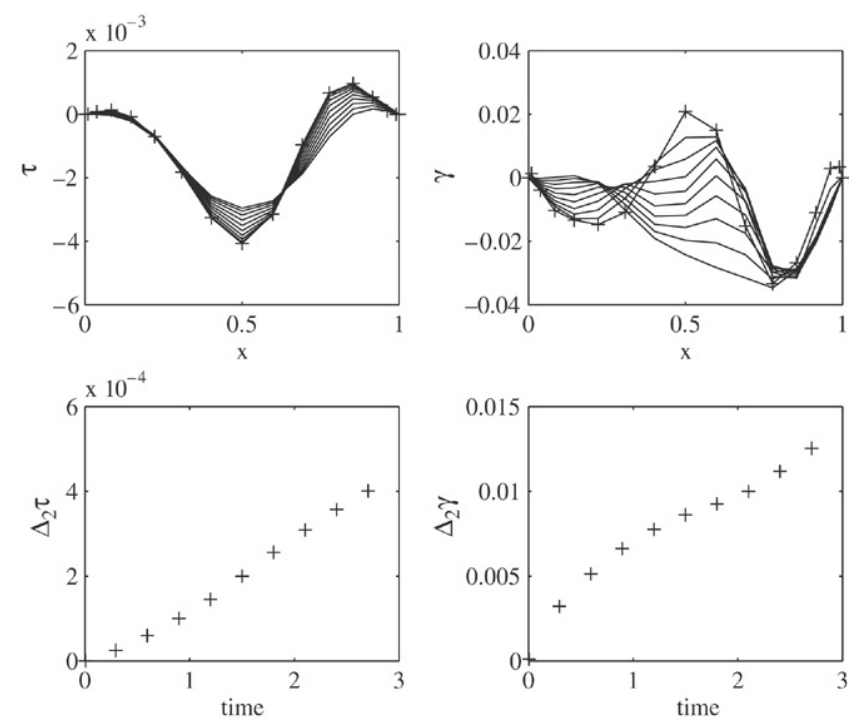

Fig. 14. Same as Fig. 13, but for the quartic RNS simulation of the lid-driven cavity flow.

Finally, we compare the local $u$-velocity reconstructed from the truncated expansion (5) using the cubic and quartic RNS simulations. Figs. 16 and 17 compare true and reconstructed $u$ velocities at select $x$ and $y$ locations near the $y=0$ boundary during the initial unsteady phase of the cavity flow.

The conclusion from figures Figs. 16 and 17 is that the skin-friction and pressure-gradient evolution obtained from the RNS equations is suitable for short-term velocity field prediction near the wall away from corners. A general discussion on velocity reconstruction from instantaneous skin-friction and wall-pressure measurements is given by Bewley and Protas [12].

\section{Conclusions}

In this paper, we have derived a hierarchy of evolution equations for two key wall-based quantities, the skin friction and the wall-pressure gradient, in two-dimensional incompressible flows. The resulting RNS equations are well-posed for smooth enough initial data. Defined over the flow boundary, the RNS equations offer reduced spatial dimensionality over the full Navier-Stokes equations; as a result, typical computation times for the RNS equations are a fraction of those for the Navier-Stokes equations.

For instance, on a $2.2 \mathrm{GHz}$ Intel Xeon processor, our spectral Navier-Stokes simulation of the lid-driven cavity flow (programmed in Fortran90) required about $1 \mathrm{~min}$ of CPU time to compute the velocity field over the convective timescale $\Delta t=0.2$. By contrast, on a slower ULTRASPARC-III $750 \mathrm{MHz}$ processor, our spectral RNS simulation of the same problem (programmed 

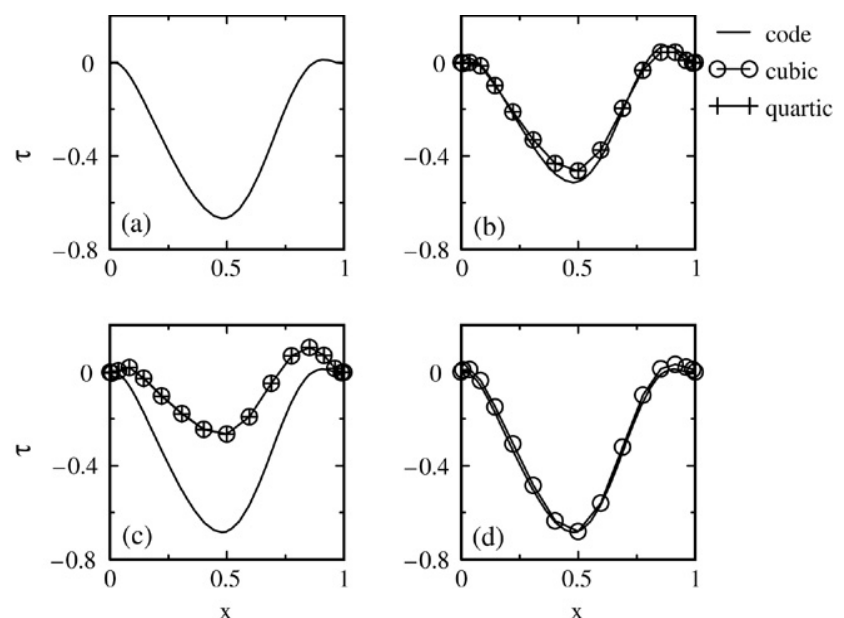

Fig. 15. Comparison of skin-friction values predicted by a Navier-Stokes simulation ("code"), and by the cubic and quartic RNS simulation for the initial unsteady phase of the lid-driven cavity flow at $R e=400$. (a) Initial condition at $\bar{t}=5.0$ (b) Simulation at $\bar{t}=5.1$ (c) simulation at $\bar{t}=5.2$ (d) Same as (c) but with initial conditions re-initialized at $\bar{t}=5.1$.
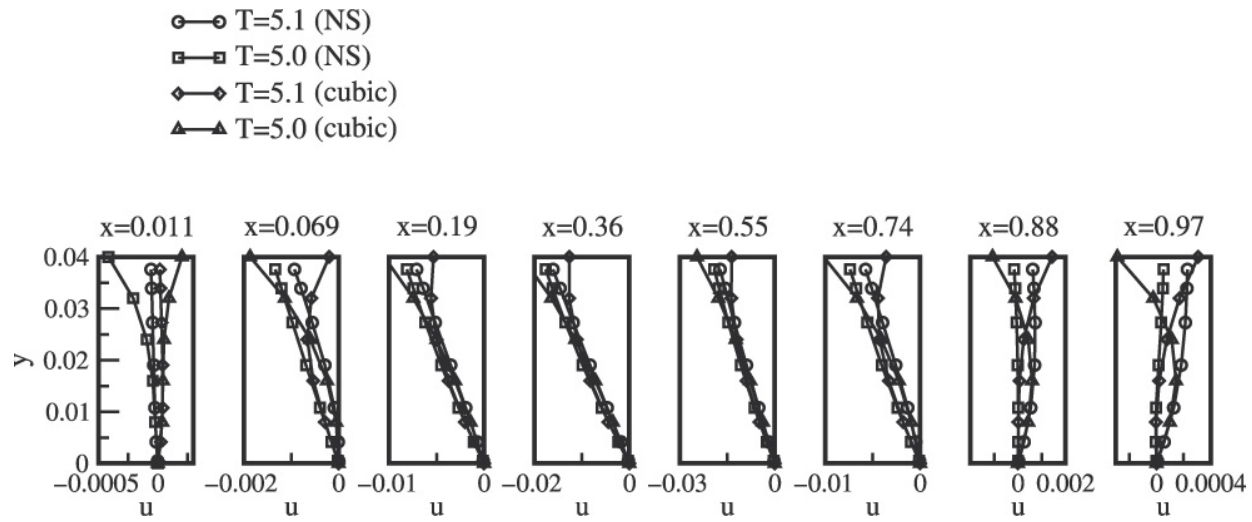

Fig. 16. The $u$-component of the lid-driven cavity velocity field at $\bar{t}=5.0$ and $\bar{t}=5.1$ at different locations near the $y=0$ boundary. "NS" refers to Navier-Stokes simulations; "cubic" refers to cubic RNS simulations.
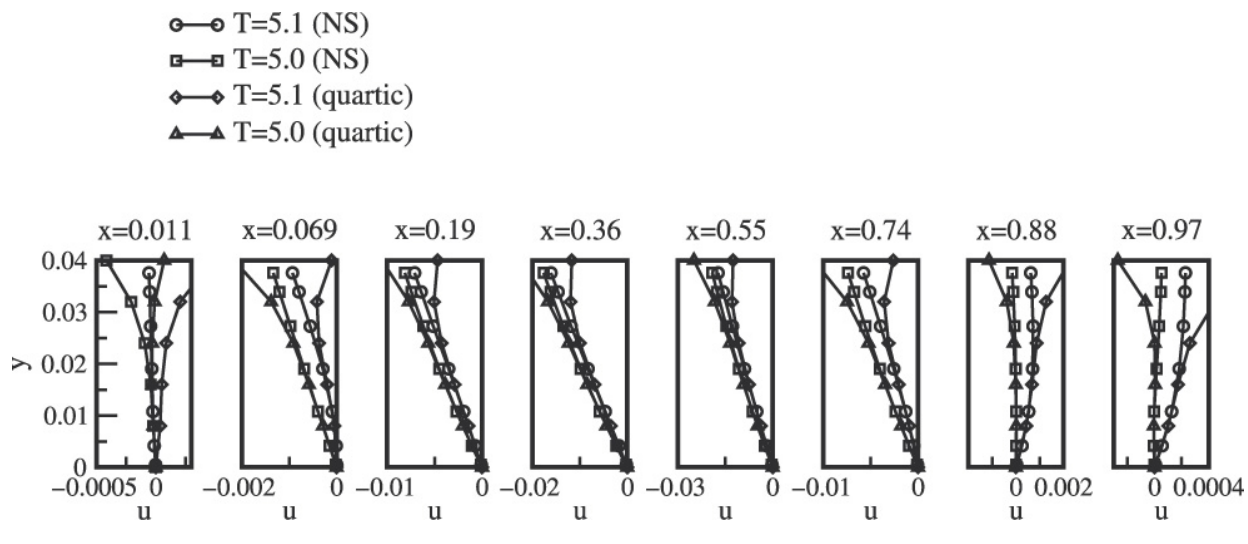

Fig. 17. Same as Fig. 16, but for the quartic RNS simulation.

in MATLAB) required only about $1.4 \mathrm{~s}$ for the cubic RNS and $1.9 \mathrm{~s}$ for the quartic RNS equation. The RNS equations, however, rely on updated boundary conditions and hence cannot be solved without observing the flow at two discrete boundary points.

Our numerical simulations on a range of benchmark problems show quantitative accuracy for short-to-intermediate times: the RNS equations produced less than $1 \%$ error over times that range from 0.1 to 3 nondimensional time units in different problems. The error growth was noticeably faster in the lid-driven cavity flow, where corner effects result in large wall-normal derivatives that are not captured by truncations of (5). In their envisioned application in flow control, however, the RNS equations should be more useful as qualitative reduced-order models rather than exact numerical tracking tools. In most of our examples, the RNS equations 
remained qualitatively accurate for times between one and 10 nondimensional time units. Over longer times, the equations require periodic re-initialization for sustained qualitative accuracy.

Our results are directly applicable in unsteady separation control when combined with the analytic approach of Alam et al. [1]. In that approach, the solution of the skin-friction equation (7) was controlled via two-point boundary actuation to satisfy the kinematic separation conditions of Haller [6]. The velocity derivative $\sigma$, however, was obtained from observations rather than from a model. We expect an improvement in the controller derived in [1] once the present RNS equations are used to obtain predictions for $\sigma$. This is explored in ongoing work.

Reduced spatial dimensionality comes at a price: higher-order RNS equations include higher-order spatial derivatives, both in the equations and in the boundary conditions. Over a certain order, the computation of derivatives becomes too expensive and the advantage of reduced spatial dimensionality is lost. For this reason, we only expect the cubic and quartic RNS equations to be effective in flow-control applications, unless the equations are posed between boundary points with known velocity derivatives.

In the present work, we have tested the RNS equations numerically for Reynolds numbers up to $10^{3}$. In this range, assuming a two-dimensional flow geometry is reasonable for a number of applications. The behavior of the RNS hierarchy for higher Reynolds numbers is expected to be more delicate. We plan to study this question in future work.

Three-dimensional extensions of the present work are possible as our initial results indicate in Section 3. In that case, the RNS equations are defined over two-dimensional boundary domains and hence require boundary conditions observed along one-dimensional curves. Such observations are possible via two-dimensional arrays of skin-friction and pressure sensors, thus applications to three-dimensional flow modelling and control appear feasible.

\section{Acknowledgements}

We thank Tom Bewley and Miroslav Krstic for useful discussions and insights. We are grateful to the anonymous referee for several helpful suggestions, including some that led to Eqs. (26) and (27). This work was supported by AFOSR Grant FA9550-061-0101 and NSF Grant DMS-04-04845. In addition, the work of G.B.J. and J.S.H. was partially supported by NSF CAREER Award DMS-01-32967.

\section{Appendix A. Well-posedness for the RNS equations}

\section{A.1. Well-posedness for the cubic RNS equations}

\section{A.1.1. Function space}

We consider the PDE system (14) with the boundary conditions (15). We will first show that (14) has a unique solution for the homogeneous boundary conditions

$$
\tau(0, t)=\tau(L, t)=0, \quad \gamma(0, t)=\gamma(L, t)=0, \quad \sigma(0, t)=\sigma(L, t)=0,
$$

on the function space

$$
X=B^{3} \times B^{2} \times B^{1},
$$

where

$$
B^{n}=\left\{u \in H^{n}([0, L]): u(0)=u(L)=0\right\} .
$$

On the space $X$, we define the norm

$$
\begin{aligned}
\|U\|^{2} & =\|(\tau, \gamma, \sigma)\|^{2}=\int_{0}^{L}\left\{|\tau|^{2}+\left|\tau_{x}\right|^{2}+\left|\tau_{x x}\right|^{2}+\left|\tau_{x x x}\right|^{2}+|\gamma|^{2}+\left|\gamma_{x}\right|^{2}+|\sigma|^{2}+\left|\sigma_{x}\right|^{2}\right\} \mathrm{d} x \\
& =\langle\tau, \tau\rangle+\left\langle\tau_{x}, \tau_{x}\right\rangle+\left\langle\tau_{x x}, \tau_{x x}\right\rangle+\left\langle\tau_{x x x}, \tau_{x x x}\right\rangle+\langle\gamma, \gamma\rangle+\left\langle\gamma_{x}, \gamma_{x}\right\rangle+\langle\sigma, \sigma\rangle+\left\langle\sigma_{x}, \sigma_{x}\right\rangle \\
& =\langle\langle\tau, \tau\rangle\rangle_{3}+\langle\langle\gamma, \gamma\rangle\rangle_{1}+\langle\langle\sigma, \sigma\rangle\rangle_{1},
\end{aligned}
$$

where $\langle\langle v, v\rangle\rangle_{n}=\langle v, v\rangle+\left\langle v_{x}, v_{x}\right\rangle+\left\langle v_{x x}, v_{x x}\right\rangle+\cdots+\left\langle\partial_{x}^{n} v, \partial_{x}^{n} v\right\rangle$.

The homogeneous boundary conditions (44) and the Eqs. (14) imply

$$
\tau_{x x}(0)=\tau_{x x}(L)=0, \quad \gamma_{x x}(0)=\gamma_{x x}(L)=0 .
$$

Differentiating the first equation in (14) twice, we obtain

$$
\tau_{x x t}=2 v \tau_{x x x x}+v \sigma_{x x},
$$


which gives

$$
\begin{aligned}
& 0=2 v \tau_{x x x x}(0)+v \sigma_{x x}(0), \\
& 0=2 v \tau_{x x x x}(L)+v \sigma_{x x}(L),
\end{aligned}
$$

by (44). Combining these last two equations with the third equation in (14) leads to

$$
\sigma_{x x}(0)=\sigma_{x x}(L)=0, \quad \tau_{x x x x}(0)=\tau_{x x x x}(L)=0 .
$$

\section{A.1.2. Evolution equation formulation}

Next we rewrite system (14) in the evolution equation form

$$
u_{t}=A u+f(u)
$$

where

$$
A=\left(\begin{array}{ccc}
2 v \partial_{x x} & 0 & v \\
0 & 2 v \partial_{x x} & 0 \\
-v \partial_{x x x x} & 0 & v \partial_{x x}
\end{array}\right), \quad f(u)=\left(\begin{array}{c}
0 \\
-\frac{1}{v \rho} \tau \tau_{x} \\
-\frac{2}{v \rho} \tau \gamma_{x}
\end{array}\right) .
$$

By a classic result of Pazy [10], the linear operator $A$ is the infinitesimal generator of a $C^{0} \operatorname{semigroup~} T(t)$ with $\|T(t)\| \leq M \mathrm{e}^{\omega t}$, if $A$ satisfies the following conditions:

(i) $A$ is closed and the domain of $A, D(A)$, is dense in $X$.

(ii) The resolvent set $\rho(A)=\left\{\lambda \in \mathbb{C}:(\lambda I-A)^{-1}\right.$ exists $\}$ of $A$ contains an interval $(\omega, \infty)$ such that for all $\lambda>\omega$, the resolvent operator

$$
R_{A}(\lambda)=(\lambda I-A)^{-1}
$$

satisfies

$$
\left\|R_{A}^{n}(\lambda)\right\| \leq M(\lambda-\omega)^{-n} .
$$

Since $A$ is a linear combination of closed differential operators, $A$ is closed. Moreover, the domain of $A$ contains $C^{\infty} \times C^{\infty} \times C^{\infty}$ which is dense in $X$.Hence, the conditions in (i) are satisfied.

To show that (ii) is also satisfied, we have to identify the spectrum of $A$. To this end, we expand $\tau, \gamma$, and $\sigma$ into Fourier series:

$$
\tau=\sum_{n=1}^{\infty} a_{n} \sin k_{n} x, \quad \gamma=\sum_{n=1}^{\infty} b_{n} \sin k_{n} x, \quad \sigma=\sum_{n=1}^{\infty} c_{n} \sin k_{n} x .
$$

(Here we have implicitly extended all three functions to the interval $[-L, 0]$ in an odd manner.) Using (47) and (48), we obtain

$$
\begin{array}{ll}
\tau_{x x}=-\sum_{n=1}^{\infty} k_{n}^{2} a_{n} \sin k_{n} x, & \tau_{x x x x}=\sum_{n=1}^{\infty} k_{n}^{4} a_{n} \sin k_{n} x, \\
\gamma_{x x}=-\sum_{n=1}^{\infty} k_{n}^{2} b_{n} \sin k_{n} x, & \sigma_{x x}=-\sum_{n=1}^{\infty} k_{n}^{2} c_{n} \sin k_{n} x,
\end{array}
$$

after integration by parts. Here the equality signs are meant in the sense of $L_{2}$ convergence; the wave number is $k_{n}=2 n \pi / L$.

By (50) and (53), the spectrum of $A$ is the union of the spectra of the three-by-three matrices

$$
A_{k_{n}}=\left(\begin{array}{ccc}
-2 v k_{n}^{2} & 0 & v \\
0 & -2 v k_{n}^{2} & 0 \\
-v k_{n}^{4} & 0 & -v k_{n}^{2}
\end{array}\right)
$$

which are found to be

$$
\sigma\left(A_{k_{n}}\right)=\left\{-2 \nu k_{n}^{2},\left(-\frac{3}{2} \pm \mathrm{i} \frac{\sqrt{3}}{2}\right) \nu k_{n}^{2}\right\} .
$$

Therefore, the spectrum of $A$ lies in the negative complex half-plane bounded away from zero. 
Although the spectrum of $A$ is confined to the negative complex half-plane, $A$ is not self-adjoint, and hence we cannot directly conclude the boundedness of $\exp (A t)$ from its boundedness in the eigenbasis. Establishing well-posedness for (49), therefore, requires more than just the boundedness of the spectrum of the linear part. For instance, the linear system

$$
\begin{aligned}
& u_{t}=A u, \\
& u(x, 0)=u_{0}(x),
\end{aligned}
$$

with $A$ defined in (50) is not well-posed on the space $\tilde{X}=L^{2} \times L^{2} \times L^{2}$, because the norm of

$$
\exp \left(A_{k_{n}} t\right)=\mathrm{e}^{-\frac{3}{2} k_{n}^{2} t}\left(\begin{array}{ccc}
\frac{2}{\sqrt{3}} \cos \left(\frac{\sqrt{3}}{2} k_{n}^{2} t+\frac{\pi}{6}\right) & 0 & \frac{2}{\sqrt{3} k_{n}^{2}} \mathrm{i} k_{n}^{-2} \sin \left(\frac{\sqrt{3}}{2} k_{n}^{2} t\right) \\
0 & \mathrm{e}^{-\frac{1}{2} k_{n}^{2} t} & 0 \\
\frac{2}{\sqrt{3}} \mathrm{i} k_{n}^{2} \sin \left(\frac{\sqrt{3}}{2} k_{n}^{2} t\right) & 0 & \frac{2}{\sqrt{3}} \cos \left(\frac{\sqrt{3}}{2} k_{n}^{2} t-\frac{\pi}{6}\right)
\end{array}\right)
$$

does not admit a $k$-independent upper bound of the form $K \mathrm{e}^{\alpha t}$ (cf. Gustaffson et al. [5]).

We proceed by defining

$$
F=(\lambda I-A) U
$$

or, in component form,

$$
\begin{aligned}
& f_{1}=\lambda \tau-2 \nu \tau_{x x}-\nu \sigma, \\
& f_{2}=\lambda \gamma-2 \nu \gamma_{x x}, \\
& f_{3}=\lambda \sigma-\nu \sigma_{x x}+\nu \tau_{x x x x} .
\end{aligned}
$$

The norm of $F$ can then be written as

$$
\begin{aligned}
\|F\|^{2}= & \left\langle\left\langle f_{1}, f_{1}\right\rangle\right\rangle_{3}+\left\langle\left\langle f_{2}, f_{2}\right\rangle\right\rangle_{2}+\left\langle\left\langle f_{3}, f_{3}\right\rangle\right\rangle_{1} \\
= & \left\langle\left\langle\lambda \tau-2 v \tau_{x x}-v \sigma, \lambda \tau-2 v \tau_{x x}-v \sigma\right\rangle\right\rangle_{3}+\left\langle\left\langle\lambda \gamma-2 v \gamma_{x x}, \lambda \gamma-2 v \gamma_{x x}\right\rangle\right\rangle_{2} \\
& +\left\langle\left\langle\lambda \sigma-v \sigma_{x x}+v \tau_{x x x x}, \lambda \sigma-v \sigma_{x x}+v \tau_{x x x x}\right\rangle\right\rangle_{1} .
\end{aligned}
$$

We find that

$$
\begin{aligned}
\left\langle\left\langle\lambda \tau-2 \nu \tau_{x x}-v \sigma, \lambda \tau-2 \nu \tau_{x x}-\nu \sigma\right\rangle\right\rangle_{3} & =\lambda^{2}\langle\langle\tau, \tau\rangle\rangle_{3}+2 \lambda \nu\left\langle\left\langle\tau,-2 \tau_{x x}-\sigma\right\rangle\right\rangle_{3}+\left\langle\left\langle 2 \tau_{x x}-\sigma, 2 \tau_{x x}-\sigma\right\rangle\right\rangle_{3} \\
& =\lambda^{2}\langle\langle\tau, \tau\rangle\rangle_{3}-2 \lambda \nu\langle\langle\tau, \sigma\rangle\rangle_{3}+4 \lambda \nu\left\langle\left\langle\tau_{x}, \tau_{x}\right\rangle\right\rangle_{3}+\left\langle\left\langle 2 \tau_{x x}-\sigma, 2 \tau_{x x}-\sigma\right\rangle\right\rangle_{3} \\
& \geq \lambda^{2}\langle\langle\tau, \tau\rangle\rangle_{3}-2 \lambda \nu\langle\langle\tau, \sigma\rangle\rangle_{3},
\end{aligned}
$$

and

$$
\begin{aligned}
\left\langle\left\langle\lambda \gamma-2 v \gamma_{x x}, \lambda \gamma-2 v \gamma_{x x}\right\rangle\right\rangle_{2} & =\lambda^{2}\langle\langle\gamma, \gamma\rangle\rangle_{2}-4 v \lambda\left\langle\left\langle\gamma, \gamma_{x x}\right\rangle\right\rangle_{2}+4 v^{2}\left\langle\left\langle\gamma_{x x}, \gamma_{x x}\right\rangle\right\rangle_{2} \\
& =\lambda^{2}\langle\langle\gamma, \gamma\rangle\rangle_{2}+4 v \lambda\left\langle\left\langle\gamma_{x}, \gamma_{x}\right\rangle\right\rangle_{2}+4 v^{2}\left\langle\left\langle\gamma_{x x}, \gamma_{x x}\right\rangle\right\rangle_{2} \\
& \geq \lambda^{2}\langle\langle\gamma, \gamma\rangle\rangle_{2} ;
\end{aligned}
$$

furthermore,

$$
\begin{aligned}
\langle\langle\lambda \sigma & \left.\left.-v \sigma_{x x}+v \tau_{x x x x}, \lambda \sigma-v \sigma_{x x}+v \tau_{x x x x}\right\rangle\right\rangle_{1} \\
& =\lambda^{2}\langle\langle\sigma, \sigma\rangle\rangle_{1}-2 \lambda \nu\left\langle\left\langle\sigma, \sigma_{x x}\right\rangle\right\rangle_{1}+2 \lambda \nu\left\langle\left\langle\sigma, \tau_{x x x x}\right\rangle\right\rangle_{1}+\left\langle\left\langle\nu \sigma_{x x}+v \tau_{x x x x}, v \sigma_{x x}+v \tau_{x x x x}\right\rangle\right\rangle_{1} \\
& \geq \lambda^{2}\langle\langle\sigma, \sigma\rangle\rangle_{1}+2 \lambda \nu\left\langle\left\langle\sigma, \tau_{x x x x}\right\rangle\right\rangle_{1}=\lambda^{2}\langle\langle\sigma, \sigma\rangle\rangle_{1}+2 \lambda \nu\left\langle\left\langle\sigma_{x x}, \tau_{x x}\right\rangle\right\rangle_{1} \\
& \geq \lambda^{2}\langle\langle\sigma, \sigma\rangle\rangle_{1}+2 \lambda \nu\left[\langle\langle\sigma, \tau\rangle\rangle_{3}-\langle\langle\sigma, \tau\rangle\rangle_{1}\right] .
\end{aligned}
$$

Using the estimates (55)-(57) in (54), we obtain

$$
\begin{aligned}
\|F\|^{2} & \geq \lambda^{2}\langle\langle\tau, \tau\rangle\rangle_{3}-2 \lambda \nu\langle\langle\tau, \sigma\rangle\rangle_{3}+\lambda^{2}\langle\langle\gamma, \gamma\rangle\rangle_{2}+\lambda^{2}\langle\langle\sigma, \sigma\rangle\rangle_{1}+2 \lambda \nu\left[\langle\langle\sigma, \tau\rangle\rangle_{3}-\langle\langle\sigma, \tau\rangle\rangle_{1}\right] \\
& =\lambda^{2}\langle\langle\tau, \tau\rangle\rangle_{3}+\lambda^{2}\langle\langle\gamma, \gamma\rangle\rangle_{2}+\lambda^{2}\langle\langle\sigma, \sigma\rangle\rangle_{1}-2 \lambda \nu\langle\langle\sigma, \tau\rangle\rangle_{1} \\
& \geq \lambda^{2}\langle\langle\tau, \tau\rangle\rangle_{3}+\lambda^{2}\langle\langle\gamma, \gamma\rangle\rangle_{2}+\lambda^{2}\langle\langle\sigma, \sigma\rangle\rangle_{1}-\lambda \nu\left[\langle\langle\sigma, \sigma\rangle\rangle_{1}+\langle\langle\tau, \tau\rangle\rangle_{1}\right] \\
& \geq\left(\lambda^{2}-\lambda \nu\right)\|U\|^{2} \geq(\lambda-v)^{2}\|U\|^{2}
\end{aligned}
$$

for all $\lambda>v$. 
Thus, for all $\lambda>v$, we obtain

$$
\frac{\|U\|}{\|F\|} \leq \frac{1}{\lambda-v} \text {. }
$$

Consequently, the resolvent $R_{A}(\lambda)$ defined in (51) satisfies

$$
\left\|R_{A}(\lambda)\right\| \leq \frac{1}{\lambda-v},
$$

or, equivalently,

$$
\left\|R_{A}^{n}(\lambda)\right\| \leq\left\|R_{A}(\lambda)\right\|^{n} \leq \frac{1}{(\lambda-v)^{n}} .
$$

We, therefore, conclude that property (52) is satisfied for $\omega \equiv v>0$, and hence $A$ is an infinitesimal generator of a $C^{0}$ semigroup $T(t)$ with

$$
\|T(t)\| \leq \mathrm{e}^{v t} .
$$

\section{A.1.3. Existence for the full cubic RNS equations}

For system (49), Pazy [10] shows that if $A$ is the infinitesimal generator of a $C^{0} \operatorname{semigroup}$ on $X$, and $f: X \rightarrow X$ is continuously differentiable, then (49) has a unique (classical) solution $u$ over the time interval $\left[t_{0}, t_{\max }\right)$. Moreover, if $t_{\max }<\infty$, then $\lim _{t \rightarrow t_{\max }}\|u(t)\|=\infty$.

We have already shown that $A$ is an infinitesimal generator of a $C^{0}$ semigroup. We now prove that $f(u)$ is continuously differentiable in $u$. We first observe that $f$ is defined and uniformly continuous on a dense subset of the complete space $X=B^{1}([0, L]) \times B^{2}([0, L]) \times B^{2}([0, L])$. As a result, $f$ can be extended continuously to the whole of $X$.

For $u=(\tau, \gamma, \sigma)$ and $h=\left(h_{1}, h_{2}, h_{3}\right) \in X$, we can write

$$
f(u+h)-f(u)=-\frac{1}{v \rho}\left(\begin{array}{c}
0 \\
h_{1} \tau_{x}+h_{1 x} \tau+h_{1} h_{1 x} \\
2\left(\tau h_{2 x}+h_{1} \gamma_{x}+h_{1} h_{2 x}\right)
\end{array}\right),
$$

which gives

$$
D_{u} f(u)=-\frac{1}{v \rho}\left(\begin{array}{cccc}
0 & 0 & 0 \\
\tau_{x}+\tau \frac{\mathrm{d}}{\mathrm{d} x} & 0 & 0 \\
2 \gamma_{x} & 2 \tau \frac{\mathrm{d}}{\mathrm{d} x} & 0
\end{array}\right),
$$

a map continuous in $X$ with respect to the norm $\|$.$\| defined in (46). Therefore, the cubic RNS equations with homogeneous$ boundary conditions are well posed: they have unique classical solutions that depend continuously on the initial conditions.

\section{A.1.4. Inhomogeneous boundary conditions}

We reconsider the system (14) with the boundary and initial conditions (15). Under the change of variables

$$
\begin{aligned}
& \tau=\tau^{\prime}+\frac{1}{L}\left[(L-x) T_{0}(t)+x T_{L}(t)\right]=\tau^{\prime}+F(x, t), \\
& \gamma=\gamma^{\prime}+\frac{1}{L}\left[(L-x) p_{x}(0, t)+x p_{x}(L, t)\right]=\gamma^{\prime}+P(x, t), \\
& \sigma=\sigma^{\prime}+\frac{1}{L}\left[(L-x) S_{0}(t)+x S_{L}(t)\right]=\tau^{\prime}+H(x, t),
\end{aligned}
$$

system (14) becomes

$$
\begin{aligned}
\tau_{t}^{\prime} & =2 v \tau_{x x}^{\prime}+v \sigma^{\prime}+v H-F_{t}, \\
\gamma_{t}^{\prime} & =2 v \gamma_{x x}^{\prime}-\frac{1}{v \rho} \tau^{\prime} \tau_{x}^{\prime}-\frac{1}{v \rho} \tau^{\prime} F_{x}-\frac{1}{v \rho} F \tau_{x}^{\prime}-\frac{1}{v \rho} F F_{x}-P_{t}, \\
\sigma_{t}^{\prime} & =v \sigma_{x x}^{\prime}-v \tau_{x x x x}^{\prime}-\frac{2}{v \rho} \tau^{\prime} \gamma_{x}^{\prime}-\frac{2}{v \rho} \tau^{\prime} P_{x}-\frac{2}{v \rho} F \gamma_{x}^{\prime}-\frac{2}{v \rho} F P_{x}-S_{t}
\end{aligned}
$$

which has a different linear part than (14) does. However, we can view (58) as

$$
u_{t}=A u+g(u) \text {, }
$$


where $A$ is given in (50) and $g$ has quadratic, linear and constant terms in $u$. It is straightforward to check that $g$ is continuously differentiable on $X$, thus all our previous arguments remain valid, and the existence of a unique solution with continuous dependence on initial data is proved.

\section{A.2. Existence and uniqueness for higher-order RNS equations}

\section{A.2.1. The linear part}

To derive the $n$th equation of the RNS system of order $N$ with $N \geq n \geq 3$, we take the $(n-3)$ th $y$-derivative of Eq. (10), then subtract from that the second $x$-derivative of the $(n-2)$ th-order RNS equation. Carrying out this procedure for only the linear parts of the equations, we obtain the linear part of the $n$ th-order RNS equation, which we explore below.

With the notation

$$
u^{n}=\rho v \frac{\partial^{n} u}{\partial y^{n}}
$$

the linear part of (10) becomes

$$
\partial_{t}\left(u^{3}+\partial_{x}^{2} u^{1}\right)=2 v \partial_{x}^{2} u^{3}+v u^{5}+\partial_{x}^{4} u^{1} .
$$

Taking the $(n-3)$ th derivative of this last equation with respect to $y$, we obtain

$$
\partial_{t}\left(u^{n}+\partial_{x}^{2} u^{n-2}\right)=2 v \partial_{x}^{2} u^{n}+v u^{n+2}+\partial_{x}^{4} u^{n-2} .
$$

This last equation is also valid for $n=1,2$, except that the terms containing $u^{n-2}$ are absent. Taking the second $x$-derivative of (59) and letting $n \rightarrow n-2$, we find that

$$
\partial_{t}\left(\partial_{x}^{2} u^{n-2}+\partial_{x}^{4} u^{n-4}\right)=2 v \partial_{x}^{4} u^{n-2}+v \partial_{x}^{2} u^{n}+\partial_{x}^{6} u^{n-4} .
$$

Subtracting (59) from (60), we obtain

$$
\partial_{t}\left(u^{n}-\partial_{x}^{4} u^{n-4}\right)=v \partial_{x}^{2} u^{n}+v u^{n+2}-\partial_{x}^{4} u^{n-2}-\partial_{x}^{6} u^{n-4} .
$$

Next, we add to (61) the equation

$$
\partial_{t}\left(\partial_{x}^{4} u^{n-4}+\partial_{x}^{6} u^{n-6}\right)=2 v \partial_{x}^{6} u^{n-4}+v \partial_{x}^{4} u^{n-2}+\partial_{x}^{8} u^{n-6},
$$

which we obtain from (59) by taking the fourth $x$-derivative and letting $n \rightarrow n-4$. The resulting equation is

$$
\partial_{t}\left(u^{n}+\partial_{x}^{6} u^{n-6}\right)=v \partial_{x}^{2} u^{n}+v u^{n+2}+\partial_{x}^{6} u^{n-4}+\partial_{x}^{8} u^{n-6},
$$

in which the order of the $y$-derivative on the left-hand side has decreased by 2 compared to (61).

We repeat the above order-reduction procedure until only $\partial_{t} u^{n}$ is left on the left-hand side of the equation. This happens when the superscript of $u$ in the second term of the left-hand side reaches -1 or -2 ; this indicates that the corresponding term is absent.

In the case of $n=4 k+i$ with $i=1,2$, the resulting linear equation is

$$
u_{t}^{n}=v \partial_{x}^{2} u^{n}+v u^{n+2}+v \partial_{x}^{n-i+2} u^{i}
$$

in the case of $n=4 k+2+i$ with $i=1,2$, the resulting linear equation is

$$
u_{t}^{n}=v u_{x x}^{n}+v u^{n+2}-v \partial_{x}^{n-i+2} u^{i} .
$$

When $n+2>N$, the term containing the superscript $n+2$ is absent, because we truncate the Taylor-expansion of $u$ at order $N$.

In summary, the linear part of the $N$ th order RNS system, say in the case of $N=4 K+1$, is of the form

$$
\begin{aligned}
& u_{t}^{1}=2 v \partial_{x}^{2} u^{1}+v u^{3}, \\
& u_{t}^{2}=2 v \partial_{x}^{2} u^{2}+v u^{4}, \\
& u_{t}^{3}=v \partial_{x}^{2} u^{3}+v u^{5}+v \partial_{x}^{4} u^{1}, \\
& u_{t}^{4}=v \partial_{x}^{2} u^{4}+v u^{6}+v \partial_{x}^{4} u^{2}, \\
& \vdots \\
& u_{t}^{4 k+i}=v \partial_{x}^{2} u^{4 k+i}+v u^{4 k+i+2}+v \partial_{x}^{4 k+2} u^{i}, \\
& u_{t}^{4 k+i+2}=v \partial_{x}^{2} u^{4 k+i}+v u^{4 k+i+2}+v \partial_{x}^{4 k+4} u^{i},
\end{aligned}
$$




$$
\begin{aligned}
& u_{t}^{4 K}=v \partial_{x}^{2} u^{4 K}+v \partial_{x}^{4 K} u^{2}, \\
& u_{t}^{4 K+1}=v \partial_{x}^{2} u^{4 K+1}+v \partial_{x}^{4 K+2} u^{1},
\end{aligned}
$$

where $i=1,2$, and all terms are evaluated at $y=0$. The structure of the RNS system for other values of $N$ is similar.

We consider the $N$ th order RNS system (63) on the function space

$$
X=B^{N} \times B^{N-1} \times \cdots \times B^{2} \times B^{1},
$$

where $B^{n}$ is defined in (45). On $X$, we define the norm

$$
\|U\|^{2}=\left\{\left\langle\left\langle u^{1}, u^{1}\right\rangle\right\rangle_{N}+\frac{1}{\sqrt{2}}\left\langle\left\langle u^{2}, u^{2}\right\rangle\right\rangle_{N-1}\right\}+\frac{1}{2}\left\{\left\langle\left\langle u^{3}, u^{3}\right\rangle\right\rangle_{N-2}+\left\langle\left\langle u^{4}, u^{4}\right\rangle\right\rangle_{N-3}\right\}+\cdots+\frac{1}{2^{(N-1) / 2}}\left\langle\left\langle u^{2 k+i}, u^{2 k+i}\right\rangle\right\rangle_{1},
$$

where $i=1$ or 2 , and

$$
\langle\langle u, v\rangle\rangle_{n}=\langle u, v\rangle+\left\langle u_{x}, v_{x}\right\rangle+\left\langle u_{x x}, v_{x x}\right\rangle+\cdots+\left\langle\partial_{x}^{n} u, \partial_{x}^{n} v\right\rangle .
$$

We observe that the homogeneous boundary conditions imply

$$
\begin{aligned}
& \partial_{x}^{2} u^{N}=0, \quad \partial_{x}^{2} u^{N-1}=0, \\
& \partial_{x}^{2 j+2} u^{N-2}=0, \quad \partial_{x}^{2 j+2} u^{N-3}=0, \quad j=0,1, \\
& \partial_{x}^{2 j+2} u^{N-4}=0, \quad \partial_{x}^{2 j+2} u^{N-5}=0, \quad j=0,1,2, \\
& \partial_{x}^{2 j+2} u^{1}=0, \quad j=0,1,2, . ., m=(N-1) / 2 .
\end{aligned}
$$

We again let

$$
F=(\lambda I-A) U,
$$

where $A$ is the linear operator appearing on the right-hand side of the RNS system (63). We estimate the norm of $F$ as

$$
\begin{aligned}
\|F\|^{2}= & \left\langle\left\langle f_{1}, f_{1}\right\rangle\right\rangle_{N}+\frac{1}{\sqrt{2}}\left\langle\left\langle f_{2}, f_{2}\right\rangle\right\rangle_{N-1}+\frac{1}{2}\left\langle\left\langle f_{3}, f_{3}\right\rangle\right\rangle_{N-2}+\cdots+\frac{1}{2^{(N-1) / 2}}\left\langle\left\langle f_{N}, f_{N}\right\rangle\right\rangle \\
= & \left\langle\left\langle\lambda u^{1}-2 v \partial_{x}^{2} u^{1}-v u^{3}, \lambda u^{1}-2 v \partial_{x}^{2} u^{1}-v u^{3}\right\rangle\right\rangle_{N}+\frac{1}{\sqrt{2}}\left\langle\left\langle\lambda u^{2}-2 v \partial_{x}^{2} u^{2}-v u^{4}, \lambda u^{2}-2 v \partial_{x}^{2} u^{2}-v u^{4}\right\rangle\right\rangle_{N-1} \\
& +\frac{1}{2}\left\langle\left\langle\lambda u^{3}-v \partial_{x}^{2} u^{3}+v \partial_{x}^{4} u^{1}-v u^{5}, \lambda u^{3}-v \partial_{x}^{2} u^{3}+v \partial_{x}^{4} u^{1}-v u^{5}\right\rangle\right\rangle_{N-2} \\
& +\frac{1}{2 \sqrt{2}}\left\langle\left\langle\lambda u^{4}-v \partial_{x}^{2} u^{4}+v \partial_{x}^{4} u^{2}-v u^{6}, \lambda u^{4}-v \partial_{x}^{2} u^{4}+v \partial_{x}^{4} u^{2}-v u^{6}\right\rangle\right\rangle_{N-3} \\
& +\frac{1}{4}\left\langle\left\langle\lambda u^{5}-v \partial_{x}^{2} u^{5}+v \partial_{x}^{6} u^{1}-v u^{7}, \lambda u^{5}-v \partial_{x}^{2} u^{5}+v \partial_{x}^{6} u^{1}-v u^{7}\right\rangle\right\rangle_{N-4} \\
& +\cdots+\frac{1}{2^{(N-1) / 2}}\left\langle\left\langle\lambda u^{N}-v \partial_{x}^{2} u^{N}+v \partial_{x}^{4} u^{N-2} \pm v \partial_{x}^{N-i+2} u^{i}, \lambda u^{N}-v \partial_{x}^{2} u^{N}+v \partial_{x}^{4} u^{N-2} \pm v \partial_{x}^{N-i+2} u^{i}\right\rangle\right\rangle_{1} \\
\geq & \lambda^{2}\left\langle\left\langle u^{1}, u^{1}\right\rangle\right\rangle_{N}+2 \lambda v\left\langle\left\langle\partial_{x} u^{1}, \partial_{x} u^{1}\right\rangle\right\rangle_{N}-2 \lambda v\left\langle\left\langle u^{1}, u^{3}\right\rangle\right\rangle_{N}+\cdots+\frac{1}{2} \lambda^{2}\left\langle\left\langle u^{3}, u^{3}\right\rangle\right\rangle_{N-2} \\
& +\lambda v\left\langle\left\langle\partial_{x} u^{3}, \partial_{x} u^{3}\right\rangle\right\rangle_{N-2}-\lambda \nu\left\langle\left\langle u^{3}, u^{5}\right\rangle\right\rangle_{N-2}+\lambda v\left\langle\left\langle\partial_{x}^{2} u^{3}, \partial_{x}^{2} u^{1}\right\rangle\right\rangle_{N-2}+\cdots+\frac{1}{4} \lambda^{2}\left\langle\left\langle u^{5}, u^{5}\right\rangle\right\rangle_{N-4} \\
& +\frac{1}{2} \lambda v\left\langle\left\langle\partial_{x} u^{5}, \partial_{x} u^{5}\right\rangle\right\rangle_{N-4}-\frac{1}{2} \lambda v\left\langle\left\langle u^{5}, u^{7}\right\rangle\right\rangle_{N-4}+\frac{1}{2} \lambda v\left\langle\left\langle\partial_{x} u^{5}, \partial_{x}^{5} u^{1}\right\rangle\right\rangle_{N-4}+\cdots \\
& \vdots \\
& +\frac{1}{2^{(N-1) / 2}}\left\{\lambda^{2}\left\langle\left\langle u^{N}, u^{N}\right\rangle\right\rangle_{1}+2 \lambda v\left\langle\left\langle\partial_{x} u^{N}, \partial_{x} u^{N}\right\rangle\right\rangle_{1}-2 \lambda v\left\langle\left\langle\partial_{x} u^{N}, \partial_{x}^{3} u^{N-2}\right\rangle\right\rangle_{1}+2 \lambda v\left\langle\left\langle\partial_{x} u^{N}, \partial_{x}^{N-i+1} u^{i}\right\rangle\right\rangle_{1}\right\} .
\end{aligned}
$$

Based on the estimates

$$
\begin{aligned}
\left|-\lambda \nu\left\langle\left\langle u^{1}, u^{3}\right\rangle\right\rangle+\lambda v_{N}\left\langle\left\langle\partial_{x}^{2} u^{3}, \partial_{x}^{2} u^{1}\right\rangle\right\rangle_{N-2}\right| & =\left|-\lambda \nu\left\langle\left\langle u^{1}, u^{3}\right\rangle\right\rangle_{1}\right| \\
& \leq \frac{1}{2} \lambda \nu\left[\left\langle\left\langle u^{1}, u^{1}\right\rangle\right\rangle_{1}+\left\langle\left\langle u^{3}, u^{3}\right\rangle\right\rangle_{1}\right],
\end{aligned}
$$




$$
\begin{aligned}
\left|\left\langle\left\langle u^{1}, u^{3}\right\rangle\right\rangle_{N}\right| & =\left|\left\langle\left\langle\partial_{x}^{2} u^{1}, \partial_{x}^{2} u^{3}\right\rangle\right\rangle_{N-2}\right|+\left|\left\langle\left\langle u^{1}, u^{3}\right\rangle\right\rangle_{1}\right| \\
& =\left|\left\langle\left\langle\partial_{x}^{3} u^{1}, \partial_{x} u^{3}\right\rangle\right\rangle_{N-2}\right|+\left|\left\langle\left\langle u^{1}, u^{3}\right\rangle\right\rangle_{1}\right| \\
& \leq \frac{1}{2}\left[\left\langle\left\langle\partial_{x}^{3} u^{1}, \partial_{x}^{3} u^{1}\right\rangle\right\rangle_{N-2}+\left\langle\left\langle\partial_{x} u^{3}, \partial_{x} u^{3}\right\rangle\right\rangle_{N-2}\right]+\left|\left\langle\left\langle u^{1}, u^{3}\right\rangle\right\rangle_{1}\right| \\
& \leq \frac{1}{2}\left[\left\langle\left\langle\partial_{x} u^{1}, \partial_{x} u^{1}\right\rangle\right\rangle_{N}+\left\langle\left\langle\partial_{x} u^{3}, \partial_{x} u^{3}\right\rangle\right\rangle_{N-2}+\left\langle\left\langle u^{3}, u^{3}\right\rangle\right\rangle_{1}\right], \\
\left\langle\left\langle\partial_{x} u^{5}, \partial_{x}^{5} u^{1}\right\rangle\right\rangle_{N-4} & \leq \frac{1}{2}\left[\left\langle\left\langle\partial_{x} u^{5}, \partial_{x} u^{5}\right\rangle\right\rangle_{N-4}+\left\langle\left\langle\partial_{x}^{5} u^{1}, \partial_{x}^{5} u^{1}\right\rangle\right\rangle_{N-4}\right] \\
& \leq \frac{1}{2}\left[\left\langle\left\langle\partial_{x} u^{5}, \partial_{x} u^{5}\right\rangle\right\rangle_{N-4}+\left\langle\left\langle\partial_{x} u^{1}, \partial_{x} u^{1}\right\rangle\right\rangle_{N}\right],
\end{aligned}
$$

we deduce the general relations

$$
\begin{aligned}
& \left\langle\left\langle u^{n}, u^{n+2}\right\rangle\right\rangle_{N+1-n} \leq \frac{1}{2}\left[\left\langle\left\langle u^{n+2}, u^{n+2}\right\rangle\right\rangle_{N-1-n}+\left\langle\left\langle u^{n}, u^{n}\right\rangle\right\rangle_{N+1-n}\right], \\
& \left\langle\left\langle\partial_{x} u^{n}, \partial_{x}^{n} u^{1}\right\rangle\right\rangle_{N+1-n} \leq \frac{1}{2}\left[\left\langle\left\langle\partial_{x} u^{n}, \partial_{x} u^{n}\right\rangle\right\rangle_{N+1-n}+\left\langle\left\langle\partial_{x} u^{1}, \partial_{x} u^{1}\right\rangle\right\rangle_{N}\right] .
\end{aligned}
$$

Combining these last two inequalities with (64), we find that for $\lambda>0$,

$$
\|F\|^{2} \geq\left(\lambda^{2}-\lambda v\right)\|U\|^{2} \geq(\lambda-v)^{2}\|U\|^{2},
$$

which again implies

$$
\left\|R_{A}^{n}(\lambda)\right\| \leq\left\|R_{A}(\lambda)\right\|^{n} \leq \frac{1}{(\lambda-v)^{n}} .
$$

Therefore, property (52) is again satisfied for the resolvent of $A$, and hence for $\omega \equiv v>0$, the linear system (63) admits a $C^{0}$ semiflow $T(t)$ with

$$
\|T(t)\| \leq \mathrm{e}^{v t} .
$$

This proves the existence, uniqueness and $C^{0}$ regularity of solutions of the $N$ th-order linearized RNS system.

\section{A.2.2. The full nonlinear system}

We now extend the above regularity result to the full $N$ th-order RNS system

$$
u_{t}=A u+f(u)
$$

where $A u$ represents the right-hand side of (63), and $f(u)$ denotes the quadratic terms. By the result of Pazy [10], system (65) is well-posed if $f: X \rightarrow X$ is continuously differentiable.

Let $\left\{\mathbf{e}_{i}\right\}_{i=1}^{N}$ be the standard basis of $\mathbb{R}^{N}$. We observe that by the definition of the space $X$, the map

$$
\begin{aligned}
& f_{l, m_{1}, n_{1}, m_{2}, n_{2}}: X \rightarrow X, \\
& u \mapsto \mathbf{e}_{l}\left(\partial_{x}^{m_{1}} u^{n_{1}}\right)\left(\partial_{x}^{m_{2}} u^{n_{2}}\right),
\end{aligned}
$$

is continuously differentiable if

$$
m_{1} \leq l-n_{1}, \quad m_{2} \leq l-n_{2} .
$$

All terms in the $N$ th-order RNS system are of the general form (66); a systematic review of these terms reveals that they all satisfy (68). (We omit a detailed listing of the nonlinear terms for brevity.)

We conclude that the function $f(u)$ in (65) is continuously differentiable, and hence the $N$ th-order RNS system with homogeneous boundary conditions admits unique solutions that are continuous with respect to the initial data. The case of inhomogeneous boundary conditions follows as in the case of the cubic RNS system (cf. Appendix A.1).

\section{Appendix B. Derivation of the cubic RNS equations in 3D}

Subtracting the $x$-derivative of the third equation in (22) from the $z$-derivative of the first equation, we obtain

$$
\begin{aligned}
& \partial_{t}\left(u_{z}-w_{x}\right)+u_{x z} u+u_{x} u_{z}+u_{y z} v+u_{y} v_{z}+u_{z z} w+u_{z} w_{z}-w_{z x} u-w_{x} u_{z}-w_{y z} v-w_{y} v_{z}-w_{z z} w-w_{z} w_{z} \\
& \quad=v\left(u_{x x z}+u_{y y z}+u_{z z z}-w_{x x x}-w_{y y x}-w_{z z x}\right) .
\end{aligned}
$$


Evaluating (69) at $z=0$ and using the no-slip boundary conditions, we obtain

$$
\left[u_{z t}\right]_{z=0}=\left[v u_{z z z}+v u_{z x x}+v u_{z y y}-v w_{z z x}\right]_{z=0} .
$$

By incompressibility, this last equation yields

$$
\left[u_{z t}\right]_{z=0}=\left[2 v u_{z x x}+v u_{z y y}+v v_{z x y}+v u_{z z z}\right]_{z=0} .
$$

The same argument applied to the $v$-component of the Navier-Stokes equations gives

$$
\left[v_{z t}\right]_{z=0}=\left[2 v v_{z y y}+v v_{z x x}+v u_{z x y}+v v_{z z z}\right]_{z=0} .
$$

We continue by taking the $z$-derivative of Eq. (69) to obtain

$$
\begin{aligned}
& \partial_{t}\left(u_{z z}-w_{z x}\right)+u_{x z z} u+2 u_{z x} u_{z}+u_{x} u_{z z}+u_{z z y} v+2 u_{z y} v_{z}+v u_{y} v_{z z}+u_{z z z} w+2 u_{z z} w_{z} \\
& +u_{z} w_{z z}-w_{z z x} u-2 w_{z x} u_{z}-w_{x} u_{z z}-w_{y z z} v-2 w_{y z} v_{z}-w_{y} v_{z z}-w_{z z z} w-3 w_{z z} w_{z} \\
& \quad=v\left(u_{z z x x}+u_{z z y y}+u_{z z z z}-w_{z x x x}-w_{z y y x}-w_{z z z x}\right) .
\end{aligned}
$$

Evaluation at $z=0$ then gives

$$
\left[u_{z z t}\right]_{z=0}=\left[-2 u_{z} u_{z x}-2 u_{z y} v_{z}-u_{z} w_{z z}+v\left(2 u_{z z x x}+u_{z z y y}+u_{z z z z}+v_{z z x y}\right)\right]_{z=0} .
$$

We use incompressibility to rewrite this last equation as

$$
\left[u_{z z t}\right]_{z=0}=\left[v\left(2 u_{z z x x}+u_{z z y y}+u_{z z z z}+v_{z z x y}\right)-u_{z} u_{z x}-2 u_{z y} v_{z}-u_{z} v_{z y}\right]_{z=0} .
$$

Similarly, we obtain

$$
\left[v_{z z t}\right]_{z=0}=\left[v\left(2 v_{z z y y}+v_{z z x x}+v_{z z z z}+u_{z z x y}\right)-v_{z} v_{z y}-2 v_{z x} u_{z}-v_{z} u_{z x}\right]_{z=0} .
$$

Differentiation of (72) with respect to $z$ gives

$$
\begin{aligned}
& \partial_{t}\left(u_{z z z}-w_{z z x}\right)+u_{z z z x} u+3 u_{z z x} u_{z}+3 u_{z x} u_{z z}+u_{x} u_{z z z}+u_{z z z y} v+3 u_{z z y} v_{z}+3 u_{z y} v_{z z} \\
& \quad+u_{y} v_{z z z}+u_{z z z} w+3 u_{z z z} w_{z}+3 u_{z z} w_{z z}+u_{z} w_{z z z}-w_{z z x} u-3 w_{z z x} u_{z}-3 w_{z x} u_{z z}-w_{x} u_{z z z} \\
& -w_{y z z z} v-3 w_{y z z} v_{z}-3 w_{y z} v_{z z}-w_{y} v_{z z z}-w_{z z z z} w-4 w_{z z z} w_{z}-3 w_{z z} w_{z z} \\
& \quad=v\left(u_{z z z x x}+u_{z z z y y}+u_{z z z z z}-w_{z z x x x}-w_{z z y y x}-w_{z z z z x}\right),
\end{aligned}
$$

which, at $z=0$, becomes

$$
\begin{aligned}
{\left[u_{z z z t}-w_{z z x t}\right]_{z=0}=} & {\left[-3 u_{z z x} u_{z}-3 u_{z x} u_{z z}-3 u_{z z y} v_{z}-3 u_{z y} v_{z z}-3 u_{z z} w_{z z}-u_{z} w_{z z z}\right.} \\
& +3 w_{z z x} u_{z}+3 w_{y z z} v_{z}+w_{z z z z} w+4 w_{z z z} w_{z}+3 w_{z z} w_{z z} \\
& \left.+v\left(u_{z z z x x}+u_{z z z y y}+u_{z z z z z}-w_{z z x x x}-w_{z z y y x}-w_{z z z z x}\right)\right]_{z=0} .
\end{aligned}
$$

Imposing incompressibility on this last equation, we find that

$$
\begin{aligned}
{\left[u_{z z z t}\right]_{z=0}=} & {\left[-u_{z x x t}-v_{z x y t}-3 u_{z z x} u_{z}-3 u_{z x} u_{z z}-3 u_{z z y} v_{z}-3 u_{z y} v_{z z}+3 u_{z z} u_{z x}\right.} \\
& +3 u_{z z} v z_{y}+u_{z} u_{z z x}+u_{z} v_{z z y}-3 u_{z x x} u_{z}-3 v_{z x y} u_{z}+3 u_{z x y} v_{z}+3 v_{z y y} v_{z}+3 u_{z x} u_{z x} \\
& +6 u_{z x} v_{z y}+3 v_{z y} v_{z y}+v\left(u_{z z z x x}+u_{z z z y y}+u_{z z z z z}-u_{z x x x x}-v_{z y x x x}-u_{z x y y x}\right) \\
& \left.-v\left(v_{z y y y x}+u_{z z z x x}+v_{z z z y x}\right)\right]_{z=0} .
\end{aligned}
$$

Using (71), we can rewrite (75) as

$$
\begin{aligned}
{\left[u_{z z z t}\right]_{z=0}=} & {\left[-v\left(2 u_{z x x x x}+u_{z y y x x}+v_{z x y x x}+u_{z z z x x}\right)-v\left(2 v_{z x y y y}+v_{z x x x y}+u_{z x x y y}+v_{z z z x y}\right)-3 u_{z z x} u_{z}-3 u_{z x} u_{z z}\right.} \\
& -3 u_{z z y} v_{z}-3 u_{z y} v_{z z}+3 u_{z z} u_{z x}+3 u_{z z} v_{z y}+u_{z} u_{z z x}+u_{z} v_{z z y}-3 u_{z x x} u_{z}-3 v_{z x y} u_{z}+3 u_{z x y} v_{z} \\
& +3 v_{z y y} v_{z}+3 u_{z x} u_{z x}+6 u_{z x} v_{z y}+3 v_{z y} v_{z y} \\
& \left.+v\left(u_{z z z x x}+u_{z z z y y}+u_{z z z z z}-u_{z x x x x}-v_{z y x x x}-u_{z x y y x}-v_{z y y y x}-u_{z z z x x}-v_{z z z y x}\right)\right]_{z=0} .
\end{aligned}
$$

After cancellations due to incompressibility, the above equation becomes

$$
\begin{aligned}
{\left[u_{z z z t}\right]_{z=0}=} & {\left[-v\left(3 u_{z x x x x}+3 u_{z y y x x}+u_{z z z x x}+3 v_{z x x x y}+3 v_{z y y y x}+2 v_{z z z x y}-u_{z z z y y}-u_{z z z z z}\right)\right.} \\
& -2 u_{z z x} u_{z}-3 u_{z z y} v_{z}-3 u_{z y} v_{z z}+3 u_{z z} v_{z y}+u_{z} v_{z z y}-3 u_{z x x} u_{z}-3 v_{z x y} u_{z}+3 u_{z x y} v_{z} \\
& \left.+3 v_{z y y} v_{z}+3 u_{z x} u_{z x}+6 u_{z x} v_{z y}+3 v_{z y} v_{z y}\right]_{z=0} .
\end{aligned}
$$


A similar argument yields

$$
\begin{aligned}
{\left[v_{z z z t}\right]_{z=0}=} & {\left[-v\left(3 v_{z y y y y}+3 v_{z y y x x}+v_{z z z y y}+3 u_{z x y y y}+3 u_{z x x x y}+2 u_{z z z x y}-v_{z z z x x}-v_{z z z z z}\right)\right.} \\
& -2 v_{z z y} v_{z}-3 v_{z z x} u_{z}-3 v_{z x} u_{z z}+3 v_{z z} u_{z x}+v_{z} u_{z z x}-3 v_{z y y} v_{z}-3 u_{z x y} v_{z}+3 v_{z x y} u_{z} \\
& \left.+3 u_{z x x} u_{z}+3 v_{z y} v_{z y}+6 v_{z y} u_{z x}+3 u_{z x} u_{z x}\right]_{z=0} .
\end{aligned}
$$

Then Eqs. (70), (71), (73), (74), (76) and (77) can be summarized as the cubic three-dimensional RNS system (24).

\section{References}

[1] M.-R. Alam, W. Liu, G. Haller, Closed-loop separation control: An analytic approach, Phys. Fluids A 18 (2006) 043601 (13 pages).

[2] C. Canuto, M.Y. Hussaini, A. Quarteroni, T. Zang, Spectral Methods in Fluid Dynamics, Springer, New York, 1987.

[3] T.J. Danielson, J.M. Ottino, Structural stability in two-dimensional model flows: Lagrangian and Eulerian turbulence, Phys. Fluids A 2 (1990) $2024-2035$.

[4] U. Ghia, K.N. Ghia, C.T. Shin, High-Re solutions for incompressible flow using the Navier-Stokes equations and a multigrid method, J. Comput. Phys. 48 (1982) 387-411.

[5] B. Gustaffson, H.-O. Kreiss, J. Oliger, Time-Dependent Problems and Difference Methods, Wiley, New York, 1995.

[6] G. Haller, Exact theory of unsteady separation for two-dimensional flows, J. Fluid Mech. 512 (2004) 257-311.

[7] C.T. Kelley, Solving Nonlinear Equations with Newton's Method, SIAM, Philadelphia, 2003.

[8] D.A. Kopriva, A staggered-grid multidomain spectral domain for the compressible Navier-Stokes equations, J. Comput. Phys. 143 (1998) 125-158.

[9] G.B. Jacobs, D.A. Kopriva, F. Mashayek, A validation study of a multidomain spectral code for simulation of turbulent flows, AIAA J. 43 (6) (2005) $1256-1264$.

[10] A. Pazy, Semigroups of Linear Operators and Applications to Partial Differential Equations, Springer, New York, 1983.

[11] A.E. Perry, M.S. Chong, A series-expansion study of the Navier-Stokes Equations with applications to three-dimensional unsteady flow, J. Fluid Mech. 173 (1986) 207-223.

[12] T.R. Bewley, B. Protas, Skin friction and pressure: The footprints of turbulence, Physica D 196 (2004) $28-44$.

[13] F.M. White, Viscous Fluid Flow, McGraw-Hill, Singapore, 1991. 